\title{
Perforin-deficient CAR T cells recapitulate late-onset inflammatory toxicities observed in patients
}

\author{
Kazusa Ishii, ${ }^{1,2,3}$ Marie Pouzolles, ${ }^{1}$ Christopher D. Chien, ${ }^{1}$ Rebecca A. Erwin-Cohen, ${ }^{4}$ M. Eric Kohler, ${ }^{1,5}$ Haiying Qin, ${ }^{1}$ \\ Haiyan Lei, ${ }^{1}$ Skyler Kuhn, ${ }^{6,7}$ Amanda K. Ombrello, ${ }^{8}$ Alina Dulau-Florea, ${ }^{9}$ Michael A. Eckhaus, ${ }^{10}$ Haneen Shalabi, ${ }^{1}$ Bonnie Yates, \\ Daniel A. Lichtenstein, ${ }^{1}$ Valérie S. Zimmermann, ${ }^{1,11}$ Taisuke Kondo, ${ }^{1}$ Jack F. Shern, ${ }^{1}$ Howard A. Young, ${ }^{4,12}$ Naomi Taylor, ${ }^{1,11}$ \\ Nirali N. Shah, ${ }^{1}$ and Terry J. Fry ${ }^{1,5}$
}

\begin{abstract}
'Pediatric Oncology Branch, Center for Cancer Research, National Cancer Institute (NCI), NIH, ${ }^{2}$ Hematology Branch, National Heart, Lung, and Blood Institute (NHLBI), NIH, and ${ }^{3}$ Experimental Transplantation and Immunotherapy Branch, Center for Cancer Research, NCI, NIH, Bethesda, Maryland, USA. ${ }^{4}$ Cancer and Inflammation Program, Center for Cancer Research, NCI, NIH, Frederick, Maryland, USA. ${ }^{5}$ Department of Pediatrics, University of Colorado Anschutz Medical Campus and Children's Hospital Colorado, Aurora, Colorado, USA. ${ }^{6}$ CCR Collaborative Bioinformatics Resource (CCBR), Center for Cancer Research, NCI, NIH, Bethesda, Maryland, USA. ${ }^{7}$ Advanced Biomedical Computational Science, Frederick National Laboratory for Cancer Research, Frederick, Maryland, USA. ${ }^{8}$ nflammmatory Disease Section, National Human Genome Research Institute, NIH, ${ }^{9}$ Department of Laboratory Medicine, NIH, and ${ }^{10}$ Diagnostic and Research Services Branch, Division of Veterinary Resources, NIH, Bethesda, Maryland, USA. "Université de Montpellier, ICMM, CNRS, Montpellier, France. 'ㄹaboratory of Cancer Immunometabolism, Center for Cancer Research, NCI, NIH, Frederick, Maryland, USA.
\end{abstract}

\begin{abstract}
Late-onset inflammatory toxicities resembling hemophagocytic lymphohistiocytosis (HLH) or macrophage activation syndrome (MAS) occur after chimeric antigen receptor T cell (CAR T cell) infusion and represent a therapeutic challenge. Given the established link between perforin deficiency and primary HLH, we investigated the role of perforin in anti-CD19 CAR T cell efficacy and HLH-like toxicities in a syngeneic murine model. Perforin contributed to both CD8 ${ }^{+}$and CD4 ${ }^{+}$CAR T cell cytotoxicity but was not required for in vitro or in vivo leukemia clearance. Upon CAR-mediated in vitro activation, perforin-deficient CAR T cells produced higher amounts of proinflammatory cytokines compared with WT CAR T cells. Following in vivo clearance of leukemia, perforin-deficient CAR T cells reexpanded, resulting in splenomegaly with disruption of normal splenic architecture and the presence of hemophagocytes, which are findings reminiscent of $H L H$. Notably, a substantial fraction of patients who received anti-CD22 CAR T cells also experienced biphasic inflammation, with the second phase occurring after the resolution of cytokine release syndrome, resembling clinical manifestations of HLH. Elevated inflammatory cytokines such as IL-1 $\beta$ and IL-18 and concurrent late CAR T cell expansion characterized the HLH-like syndromes occurring in the murine model and in humans. Thus, a murine model of perforin-deficient CAR T cells recapitulated late-onset inflammatory toxicities occurring in human CAR T cell recipients, providing therapeutically relevant mechanistic insights.
\end{abstract}

\section{Introduction}

Chimeric antigen receptor T cells (CAR T cells) are highly effective against $\mathrm{B}$ cell malignancies (1-8) but are frequently associated with cytokine-mediated toxicities or hyperinflammatory conditions $(9,10)$. The most well-described cytokine-mediated toxicity after CAR T cell treatment is cytokine release syndrome (CRS), which typically occurs during initial CAR T cell expansion and is associated with marked elevation of multiple inflammatory cytokines (2, 9-13). Patients present with a constellation of signs and symptoms resembling systemic inflammatory response syndrome or sepsis, in the absence of any identifiable infectious etiology. IL-6 has been recognized as an important mediator of CRS (2, 9, $12,14)$, and management of CRS with tocilizumab, an anti-IL-6

Authorship note: NNS and TJF are co-senior authors. MP and CDC are co-second authors.

Conflict of interest: The authors have declared that no conflict of interest exists. Copyright: ( 2020, American Society for Clinical Investigation.

Submitted: May 30, 2019; Accepted: July 9, 2020; Published: September 14, 2020

Reference information: J Clin Invest. 2020;130(10):5425-5443.

https://doi.org/10.1172/JCl130059. receptor monoclonal antibody $(9,10,12)$, has been highly effective, leading to its FDA approval for the treatment of CAR T cell-related CRS (15). A prolonged life-threatening systemic inflammatory response resembling hemophagocytic lymphohistiocytosis (HLH) or macrophage activation syndrome (MAS) (10) has been increasingly recognized as one part of the spectra of toxicities occurring after anti-CD19 CAR T cell infusion or CD19-directed blinatumomab, a bispecific T cell engager therapy (14). HLH-like manifestations have also been seen after anti-CD22 CAR T cell therapy (16). HLH and MAS are clinical syndromes of pathologic hyperinflammation and uncontrolled macrophage activation associated with triggers such as viral infections and rheumatologic diseases $(17,18)$. Similarly, patients with CAR T cell-associated HLH-like toxicities present with prolonged cytopenia, hemophagocytosis, hyperferritinemia, fever, coagulopathy, liver function abnormalities, splenomegaly, and other organ dysfunction $(14,16)$.

The pathophysiology of CAR T cell-induced toxicities is poorly understood, largely because of the lack of experimental models. In particular, currently available xenograft murine models are unsuitable for addressing interactions between CAR T cells and host immune cells because of confounding xenogeneic 
A
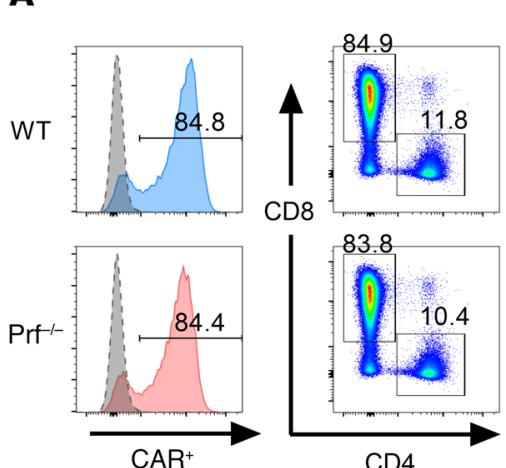

C

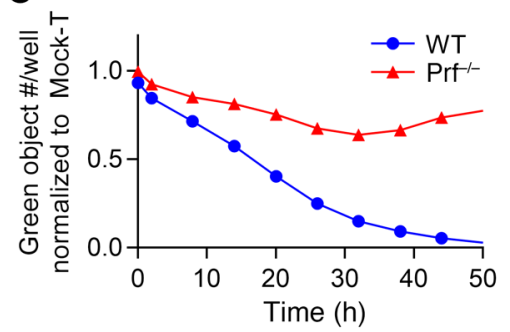

$\mathbf{F}$
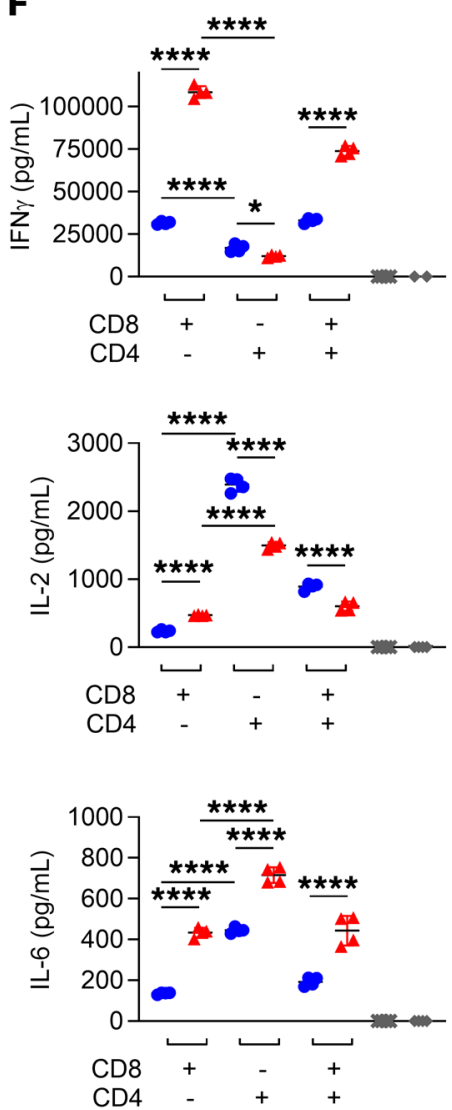

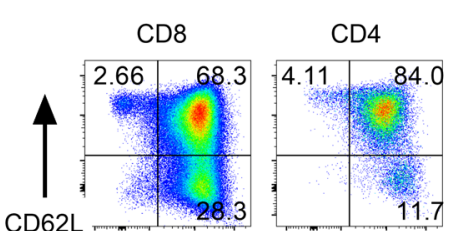

B

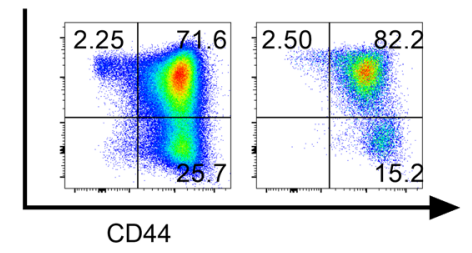

D

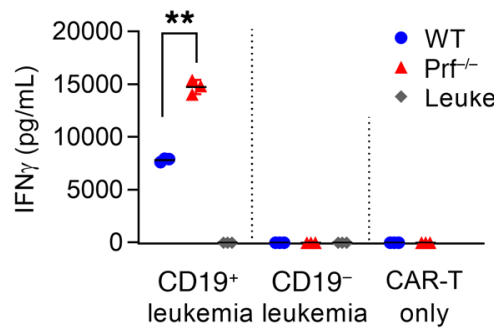
leukemia leukemia only

E
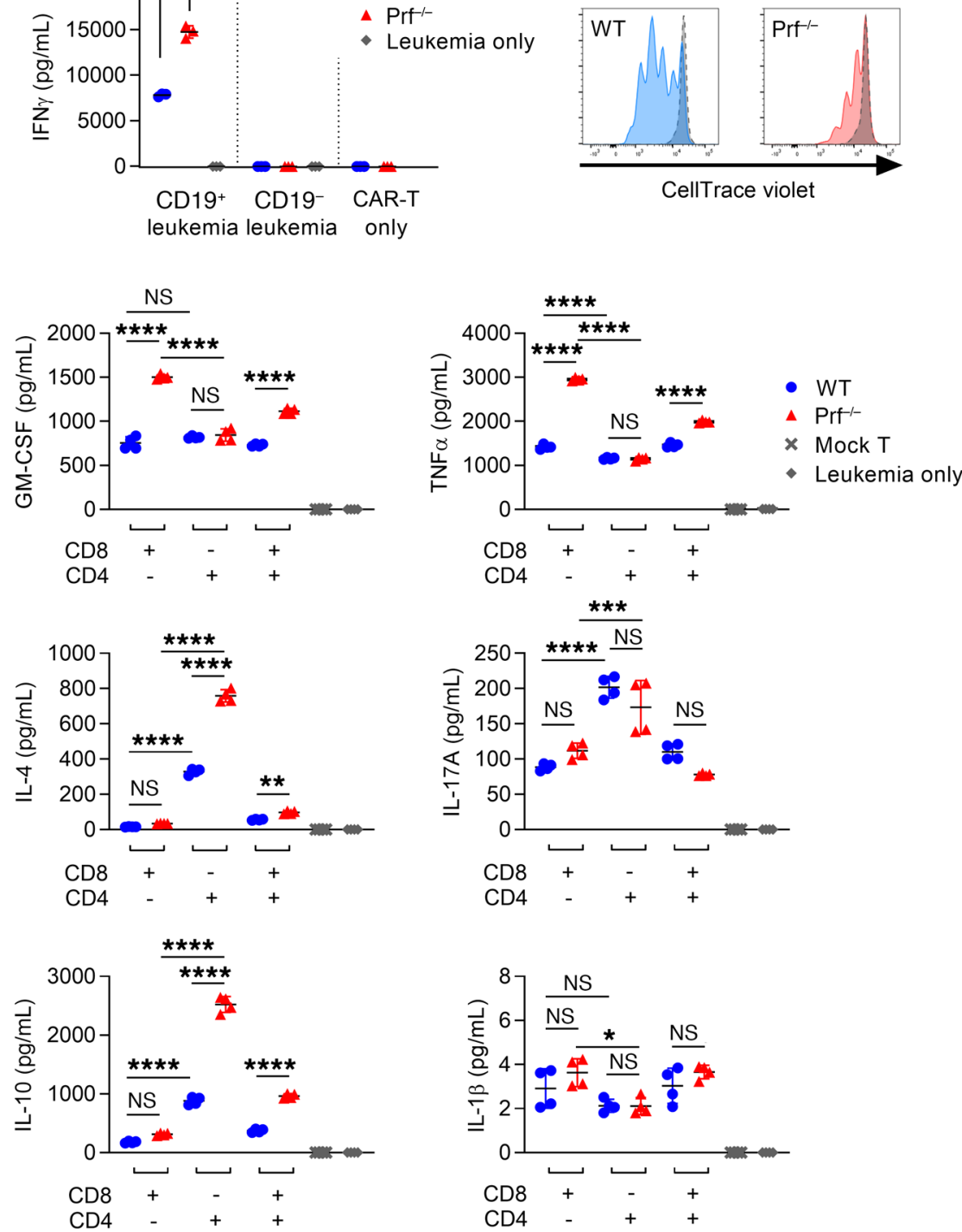
Figure 1. Prf ${ }^{-1-}$ CAR T cells produce increased proinflammatory cyto-

kines. (A) Cell product characterization: cells were stained for surface CAR expression, CD4/CD8, and CD62L/CD44 and analyzed by flow cytometry 48 hours after the completion of CAR transduction. Gray-dotted histogram overlays represent the untransduced T cell control. (B) CD107a expression on CAR T cells after 4 hours of incubation with CD19+ or CD19- E2aPBX cells. Representative histogram shows CD107a expression on CAR T cell after stimulation with CD19+ E2aPBX cells; gray-dotted histogram overlays represent the isotype control. (C) In vitro cytotoxicity measured by IncuCyte Zoom: GFP-transduced E2aPBX cells were cocultured with CAR T cells (E:T $=2: 1)$. Green objects (GFP+ leukemia cells) were counted at each time point and normalized to untransduced T cell wells $(n=2$, biological duplicate). AUC for Prf $^{-1-}$ CAR T cell was 37.8 (95\% Cl: 37.6-38.1). AUC for WT CAR T cells was 17.8 ( $95 \% \mathrm{Cl}: 17.6-18.0)$. (D) IFN- $\gamma$ levels in the 12 -hour coculture supernatant of CAR T cells with CD19+ or CD19- E2aPBX cells (E:T $=1: 1$ ), as measured by ELISA. (E) Proliferation assay: CAR T cells labeled with CellTrace Violet were cocultured with either CD19+ or CD19- E2aPBX cells $(E: T=1: 1)$ for 3 days and analyzed by flow cytometry. Gray-dotted histogram overlays represent CAR T cells incubated with CD19- E2aPBX cells (unstimulated controls). Representative histograms from 3 biological replicates are shown. (F) CD4+ CAR T cells, CD8 ${ }^{+}$CAR T cells, or $\mathrm{CD} 4^{+}$and $\mathrm{CD}^{+}$CAR T cells (1:1 mixture) were cocultured with E2aPBX cells overnight $(E: T=1: 1)$. Cytokine levels were measured in the coculture supernatant using the Meso Scale Discovery U-PLEX kit. Data are reported as the mean $\pm \mathrm{SD}$ (B, D, and F). $n=3$ (B and $\mathbf{D}) ; n=4-5(\mathbf{F})$. Figures are representative of 3 replicate experiments. ${ }^{*} P<0.05,{ }^{* *} P<0.01,{ }^{* *} P<0.001$, and ${ }^{* * *} P<$ 0.0001 , by Kruskal-Wallis test with Dunn's correction (B and $\mathbf{D}$ ) and 1-way ANOVA with Šidák's correction (F).

graft-versus-host effects, lack of cross-reactivity of certain cytokines between human and murine receptors, and the immunodeficient mice used as recipients. One subset of primary, or familial, HLH occurs in individuals harboring genetic defects in granule-mediated cytotoxic pathways, such as perforin gene mutations $(17,19,20)$. In a well-studied primary HLH murine model in which perforin-deficient mice are infected with lymphocytic choriomeningitis virus (LCMV), IFN- $\gamma$ derived from antigen-specific $\mathrm{T}$ cells has been implicated as a major mediator of HLH pathology $(21,22)$. The role of perforin in CAR T cells has not been well studied, and it is unknown whether HLH-like secondary inflammatory conditions can be triggered by CAR T cells with low or absent levels of perforin.

Here, we examined the role of granule-mediated cytotoxicity in CAR T cell responses and toxicity by evaluating the function of perforin-deficient CAR $\mathrm{T}$ cells in a syngeneic murine model of anti-CD19 CAR T cell therapy against pre-B cell acute lymphoblastic leukemia (pre-B ALL) (23-26). We found that perforin was not required for leukemia clearance by CAR T cells but was critical for CAR T cell cytotoxic potency. Under conditions of perforin deficiency, CAR T cells underwent reexpansion, and recipient mice developed a hyperinflammatory response characterized by HLH-like manifestations in the absence of detectable target antigen. Importantly, a similar biphasic inflammation and CAR T cell kinetics - CRS and the resolution thereof, followed by HLH-like manifestations - were observed in a subset of patients receiving anti-CD22 CAR T cells for B cell malignancies (ClinicalTrials.gov NCT02315612). Finally, elevation of IL-1 $\beta$ and IL-18 in both mice and patients with HLH-like manifestations linked these cytokines to the clinical phenotype, indicating potential therapeutic strategies.

\section{Results}

Perforin-deficient CAR T cells produce increased proinflammatory cytokines. WT and Prf1-KO ( Prf $\left.^{-1}\right)$ T cells were transduced with a murine anti-CD19 CAR harboring a CD28 costimulatory domain (27). WT and Prf ${ }^{-/}$CAR T cells demonstrated equivalent transduction efficiency, CD4/CD8 ratios, and central memory (Tcm)/ effector memory (Tem) or effector $\mathrm{T}$ (Teff) cell composition (Figure 1A). Both WT and Prf $^{-/}$CAR T cells upregulated CD107a in response to stimulation with $\mathrm{CD} 19^{+}$leukemia (Figure 1B), providing evidence that CAR $\mathrm{T}$ cell degranulation is not dependent on perforin, which is consistent with the activation of T cells and NK cells through physiologic receptors $(21,28)$. However, Prf $^{-/}$CAR T cells demonstrated inferior in vitro cytotoxicity with slower kinetics of leukemia clearance compared with WT CAR T cells (Figure 1C). Interestingly, Prf $^{-/}$CAR T cells produced significantly higher levels of IFN- $\gamma$ than did WT CAR T cells (Figure 1D) but showed less proliferation in response to CAR-mediated stimulation (Figure 1E). The decreased proliferative capacity of $\mathrm{Prf}^{-/-}$CAR T cells was not due to high levels of IFN- $\gamma$, because a neutralizing antiIFN- $\gamma$ monoclonal antibody did not improve proliferation (Supplemental Figure 1; supplemental material available online with this article; https://doi.org/10.1172/JCI130059DS1). Analysis of cytokine production by purified $\mathrm{CD} 4^{+}$and $\mathrm{CD} 8^{+} \mathrm{CAR} \mathrm{T}$ cells (Supplemental Figure 2) indicated that the majority of IFN- $\gamma$ was secreted by $\mathrm{CD}^{+}$CAR T cells (Figure $1 \mathrm{~F}$ ). In addition to IFN- $\gamma, \mathrm{Prf}^{-/-} \mathrm{CAR}$ $\mathrm{T}$ cells produced significantly higher amounts of other proinflammatory cytokines such as granulocyte macrophage CSF (GM-CSF) and TNF- $\alpha$ compared with WT CAR T cells (Figure 1F).

Next, we performed gene expression profiling to explore differentially expressed genes in CAR T cells with or without perforin. Comparison of unstimulated WT and $\mathrm{Prf}^{-/-} \mathrm{CD} 8^{+}$CAR T cells ( 8 days after the initial $\mathrm{T}$ cell activation and 4 days after the removal of Dynabeads Mouse T-Activator CD3/CD28) identified 117 genes that were up- or downregulated by more than 2-fold. Genes involved in inflammation were not differentially expressed in unstimulated Prf $^{--}$CAR T cells or WT CAR T cells, and pathway analysis showed an enrichment for the biological process of "cytolysis" (GO: 0019835, $P$ value $1.1 \times 10^{-7}$ ). Following stimulation of WT and $\mathrm{Prf}^{-/} \mathrm{CD} 8^{+}$CAR T cells with CD19+ leukemia cells for 24 hours, a total of 226 genes were up- or downregulated by more than 2 -fold. In contrast to the pathway analysis of the unstimulated CAR T cells, this comparison showed an enrichment for "immune response" (GO:0006955, $P$ value $9.1 \times 10^{-22}$ ) and "inflammatory response" (GO:0006954, $P$ value $4.5 \times 10^{-13}$ ) pathways, consistent with the significantly higher in vitro secretion of proinflammatory cytokines by $\mathrm{Prf}^{-/-} \mathrm{CD}^{+} \mathrm{CAR} \mathrm{T}$ cells (Figure 1, $\mathrm{D}$ and F). Upregulated genes in $\mathrm{Prf}^{-/-} \mathrm{CD} 8^{+} \mathrm{CAR} \mathrm{T}$ cells included multiple proinflammatory cytokines and chemokines such as $I l 13$ (14.4-fold), Csf2 (GM-CSF, 8.7-fold), Il5, (4.6-fold), Cxcl9 (4.5-

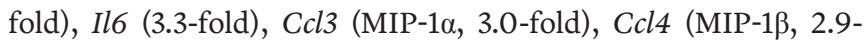
fold), Cxcl1O (2.5-fold), and Il1a (2.5-fold) as well as regulatory molecules associated with activated T cells (Ctla4, 4.5-fold; Lag3, 1.9-fold) (Supplemental Figure 3 and Supplemental Table 3). Interestingly, although most IL-1 family cytokines, such as IL-1 $\beta$ and IL-18, which are predominantly derived from non-T cell populations, were not differentially expressed, an IL-1 $\beta$-inducible gene, Tnfaip3, was significantly upregulated (2.2-fold) in $\mathrm{Prf}^{-/-}$CAR 
A

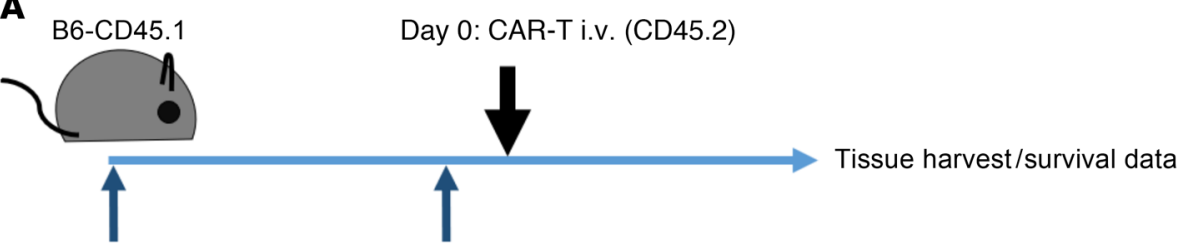

Day -6: E2aPBX (pre-B ALL, Day -1: Cyclophosphamide 200 mg/kg i.p.

CD45.2), 1 E6 i.v.

B

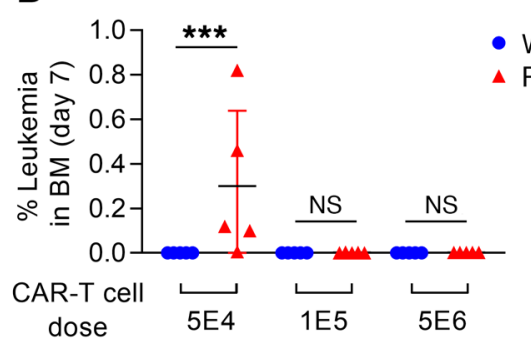

D

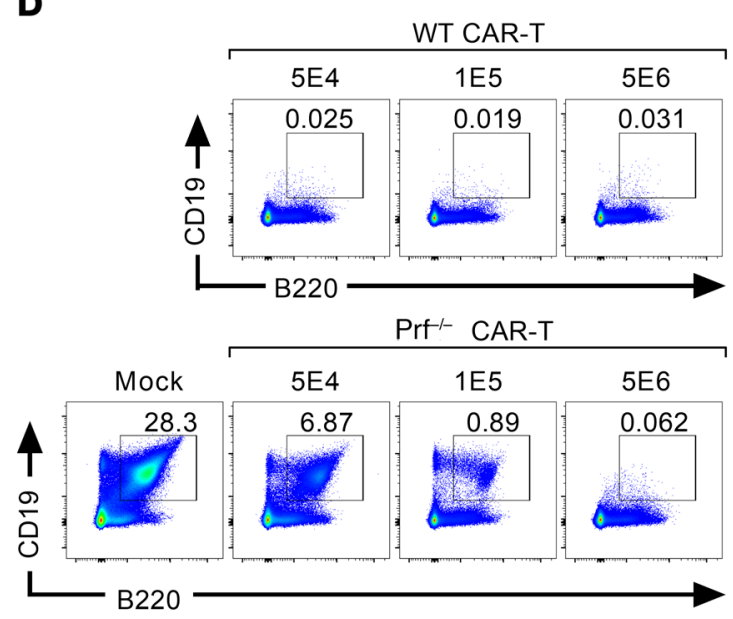

G

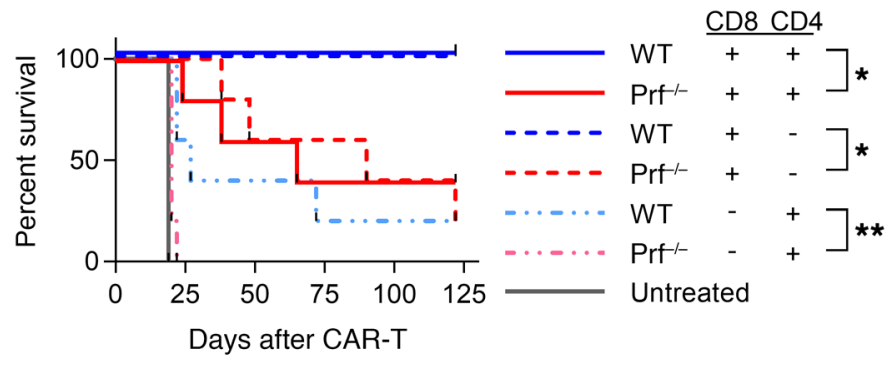

\section{C}

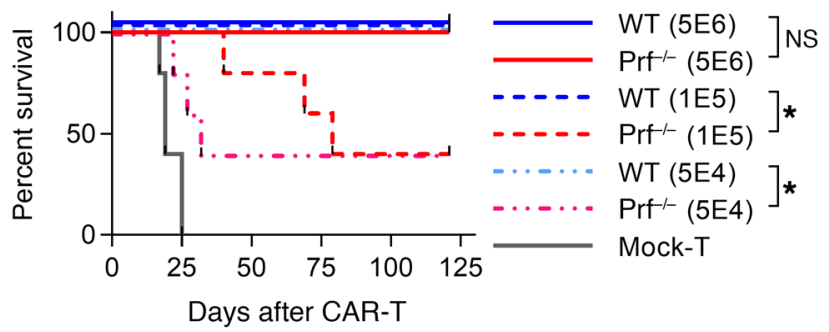

E

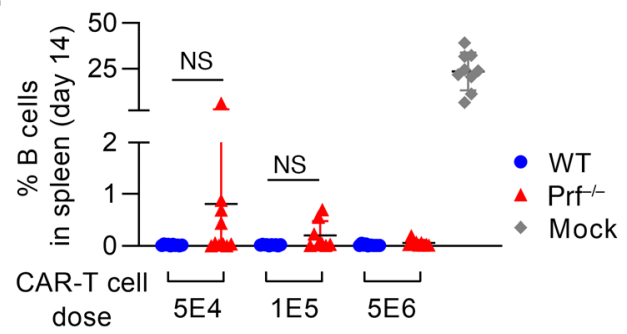

$\mathbf{F}$

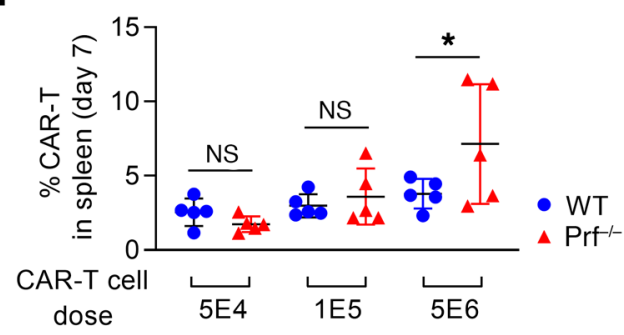

H

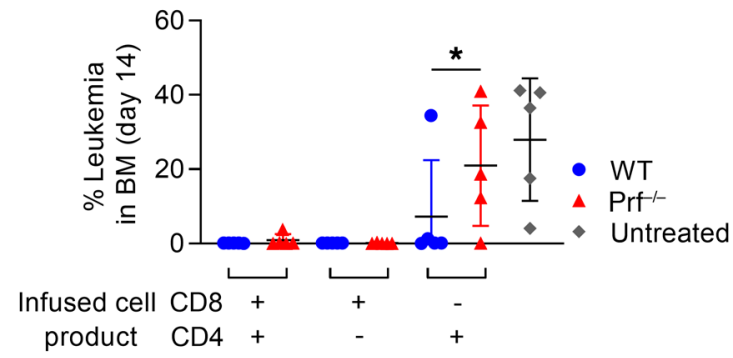


Figure 2. Prf--- CAR T cells exhibit inferior cytotoxicity compared with WT CAR T cells. (A) In vivo treatment scheme: B6-CD45.1 mice were injected with $1 \times 10^{6}$ E2aPBX (CD45.2+) cells via tail vein injection (i.v.) on day -6 , lymphodepleted with cyclophosphamide i.p. injection $(200 \mathrm{mg} / \mathrm{kg})$ on day -1 , and administered CAR T cells (CD45.2+) i.v. on day 0. (B-F) Leukemia-bearing B6-CD45.1 mice were treated with either WT or Prf ${ }^{-1-}$ CAR T cells at a cell dosage of $5 \times 10^{4}, 1 \times 10^{5}$, or $5 \times 10^{5}$. (B) BM leukemia burden (CD45.2+CD19+) on day 7 was assessed by flow cytometry. (C) Kaplan-Meier survival curve. (D and E) Total splenic B cells (CD19+B220+) on day 14 were assessed by flow cytometry. (D) Representative dot plots and (E) statistical comparisons are shown. (F) Adoptively transferred $T$ cells (CD45.2 $2^{+}$and either $C D 8^{+}$or $C D 4^{+}$) in spleens on day 7 were assessed by flow cytometry. ( $\mathbf{G}$ and $\mathbf{H}$ ) CAR T cells were manufactured from $\mathrm{CD} 4^{+}$or CD8 ${ }^{+}$purified splenic T lymphocytes. Leukemia-bearing B6-CD45.1 mice were treated with either $\mathrm{CD}^{+}, \mathrm{CD} 8^{+}$, or a $1: 1$ mixture of $\mathrm{CD} 4^{+}$and $\mathrm{CD} 8^{+} \mathrm{CAR}$ T cells manufactured from WT or Prf ${ }^{-1-}$ donors (total CAR T cells: $1 \times 10^{5}$ cells/mouse), according to the experimental scheme depicted in $\mathbf{A}$. (G) Kaplan-Meier survival curve and (H) BM leukemia burden (CD45.2+CD19+) on day 14 , as assessed by flow cytometry. Data are reported as the mean $\pm \mathrm{SD}(\mathbf{B}, \mathbf{E}, \mathbf{F}$, and $\mathbf{H}) . n=5$ (B, C, F, G, and $\mathbf{H}) ; n=10$ (E, pooled data from 2 independent experiments). Figures are representative of 2 replicate experiments. ${ }^{*} P<0.05,{ }^{* *} P<0.01$, ${ }^{* *} P<0.001$, and ${ }^{* * *} P<0.0001$, by Kruskal-Wallis test with Dunn's correction (B, E, and $\mathbf{H}), 1$-way ANOVA with Šidák's correction (F), or log-rank (Mantel-Cox) test (C and $\mathbf{G}$ ).

T cells. Furthermore, IL-1 $\beta$-induced cytokines such as $I l 22$ and Il24 (29) were upregulated by 4.4 -fold and 3.5-fold, respectively. Finally, expression levels of Il18rap and Il18ra were significantly lower in Prf $^{-/-}$CAR T cells ( -2.3 fold and -2.4 fold, respectively) compared with expression in WT CAR T cells. Collectively, these results suggest that perforin-deficient CAR T cells produce significantly higher levels of multiple proinflammatory mediators after antigen encounter compared with their WT counterpart.

Perforin contributes to, but is not required for, CAR T cell-mediated leukemia clearance. We next evaluated the role of perforin in CAR T cell expansion and in vivo antileukemia cytotoxicity (Figure $2 \mathrm{~A}$ ). Consistent with the differences we detected in in vitro cytotoxicity (Figure 1C), $\operatorname{Prf}^{-/}$CAR T cells were less efficient at leukemia clearance than were WT CAR T cells, although this difference could be overcome with a higher cell dose (Figure 2, B and C). We also detected reduced cytotoxic potency of $\operatorname{Prf}^{-/}$CAR T cells as incomplete B cell aplasia at low doses (Figure 2, D and E). Despite impaired in vitro proliferation in the absence of perforin (Figure 1E), we found that early in vivo CAR T cell expansion was comparable between $\mathrm{Prf}^{-/}$and WT CAR T cells at low doses and even superior in Prf $^{-/-}$CAR T cells at high doses (Figure 2F), indicating that treatment failure of low-dose $\mathrm{Prf}^{-/}$CAR T cells was not due to poor in vivo CAR T cell expansion. Lack of perforin negatively affected the cytotoxicity of both $\mathrm{CD} 8^{+}$and $\mathrm{CD} 4^{+} \mathrm{CAR}$ T cells (Figure 2G and Supplemental Figure 4A). This deficit was particularly pronounced in mice receiving $\mathrm{Prf}^{-/-} \mathrm{CD} 4^{+} \mathrm{CAR} \mathrm{T}$ cells (Figure $2 \mathrm{H}$ ), whose survival was similar to that of mock T cell-recipient mice (Figure $2 \mathrm{G}$ ), despite an initial expansion that was comparable to that seen in WT CD4 ${ }^{+}$CAR T cells (Supplemental Figure 4, B and $\mathrm{C}$ ). Thus, perforin contributes to the antileukemia function of both $\mathrm{CD}^{+}$and $\mathrm{CD} 4^{+} \mathrm{CAR}$ T cells.

Prf/- CAR T cells undergo a secondary expansion with activated phenotypes in the absence of detectable antigens. Interestingly, the difference in engrafted $\operatorname{Prf}^{-/}$CAR $\mathrm{T}$ cell versus WT CAR T cell frequencies was even greater on day 14 than day 7 , most notably in the $\mathrm{CD}^{+}$fraction when given at high doses $\left(5 \times 10^{6}\right)$ (Figure $3 \mathrm{~A}$ and Supplemental Figure 5A). Despite comparable transduction efficiency at the time of adoptive $\mathrm{T}$ cell transfer (Figure 1A), higher numbers of adoptively transferred $\mathrm{Prf}^{-/} \mathrm{CD}^{+} \mathrm{T}$ cells expressed surface CAR compared with WT on day 14 (Figure 3B). These data suggest either an enhanced expansion and/or persistence of CAR-expressing cells or an attenuated downregulation of CAR expression at the cell surface in Prf $^{-/-}$CAR T cells. Next, we evaluated the kinetics of CAR T cell expansion and phenotypes concurrently with the kinetics of antigen burden (Figure 3, C-F). Both WT and $\mathrm{Prf}^{-/}$CAR T cells eradicated CD19 ${ }^{+}$leukemia cells (Figure 3C) and normal B cells (Figure 3D) with similar kinetics at high doses $\left(5 \times 10^{6}\right)$. CD19-expressing cells were cleared during the first 4 to 5 days after CAR T cell infusion, corresponding to the timing of initial maximal in vivo expansion (Figure $3 \mathrm{E}$ ). CAR T cells then contracted by day 8 . However, Prf ${ }^{--}$CAR T cells, but not WT CAR T cells, reexpanded between days 13 and 20 in the absence of detectable CD19+ leukemia or normal B cells in bone marrow (BM) or spleen (Figure 3, C-E). Furthermore, we detected surface CAR expression on the majority of $\mathrm{Prf}^{-/-} \mathrm{CD} 8^{+}$CAR T cells during this reexpansion phase, whereas the levels on WT CAR T cells decreased (Figure 3, F and $\mathrm{G})$. This change in CAR expression was associated with a significantly higher percentage of effector (Teff or Tem, CD $44^{+} \mathrm{CD} 62 \mathrm{~L}^{-}$) cells within the CD8 $8^{+}$subset of $\mathrm{Prf}^{-/}$CAR T cells as compared with WT CAR T cells (Figure 3, H and I). Conversely, the percentage of Tcm cells $\left(\mathrm{CD} 44^{+} \mathrm{CD} 62 \mathrm{~L}^{+}\right)$was significantly lower in the $\mathrm{Prf}^{-/}$ CD ${ }^{+}$CAR $\mathrm{T}$ cell subset by 8 days after adoptive transfer (Figure 3J). Prf $^{-/} \mathrm{CD}^{+} \mathrm{CAR} \mathrm{T}$ cells also expressed significantly higher levels of activation-induced inhibitory markers including PD-1, TIM3, and LAG3 as compared with WT CAR T cells (Figure 3, K-N). Within the $\mathrm{CD}^{+}$subset, $\mathrm{Prf}^{-/}$CAR T cells also showed similar trends of late in vivo CAR T cell reexpansion and phenotypic terminal differentiation (Supplemental Figure 5, A-H). In summary, the lack of perforin resulted in a reexpansion of activated CAR T cells, even without a detectable antigen.

Prf $^{\prime-}$ CAR T cells induce secondary inflammatory changes that phenotypically resemble $H L H$. During the reexpansion phase (between days 8 and 20), Prf $^{-/}$CAR T cell recipients developed a marked splenomegaly with an increase in absolute splenocyte counts of more than 2- to 3-fold (Figure 4, A and B). Histologically, spleens in the mice adoptively transferred with $\mathrm{Prf}^{-/-}$CAR T cells had poorly demarcated white pulp expansion (Figure 4C, bottom left panel) with a "starry sky" appearance, representing phagocytes that have engulfed apoptotic cells and surrounding lymphocytic infiltration (Figure 4C, bottom middle and bottom right panels; phagocytes are highlighted with yellow arrows and shown at 100 $\times$ magnification in an inset). They were further characterized by increased mitotic figures (Figure 4C, bottom right panel, highlighted with red arrows) and the presence of hemophagocytes (Figure 4D). This was in contrast to WT CAR T cell-recipient mice, in which normal splenic architecture with concentric white pulps was preserved without evidence of increases in phagocytic cells or mitoses (Figure 4C, top panel). The adoptive transfer of $\mathrm{Prf}^{-/}$ CAR $\mathrm{T}$ cells also resulted in the proportionate expansion of recipient-derived immune cells, with an increase in absolute numbers of recipient-derived $\mathrm{CD} 8^{+} \mathrm{T}$ cells and $\mathrm{CD} 11 \mathrm{~b}^{+}$myeloid cells compared with numbers observed in WT CAR T cell recipients (Fig- 
A

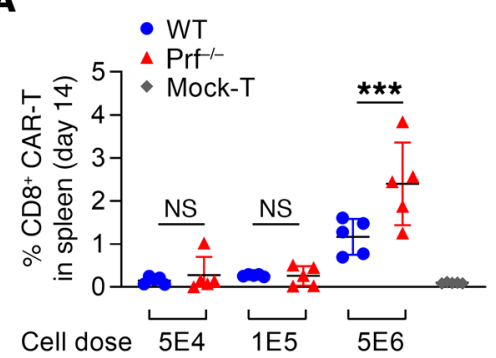

C

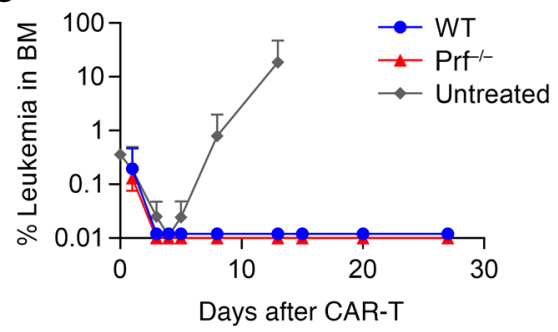

$\mathbf{F}$

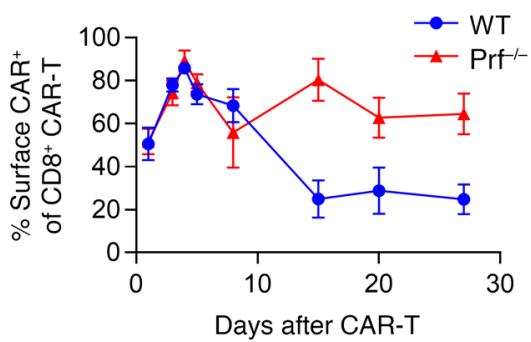

B

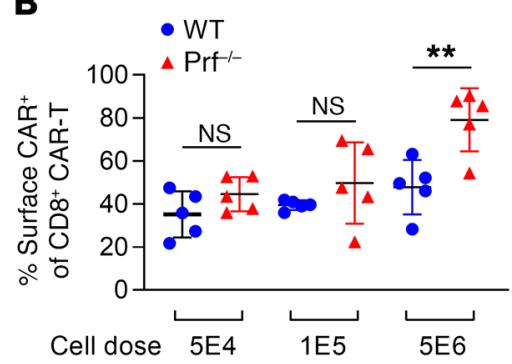

D
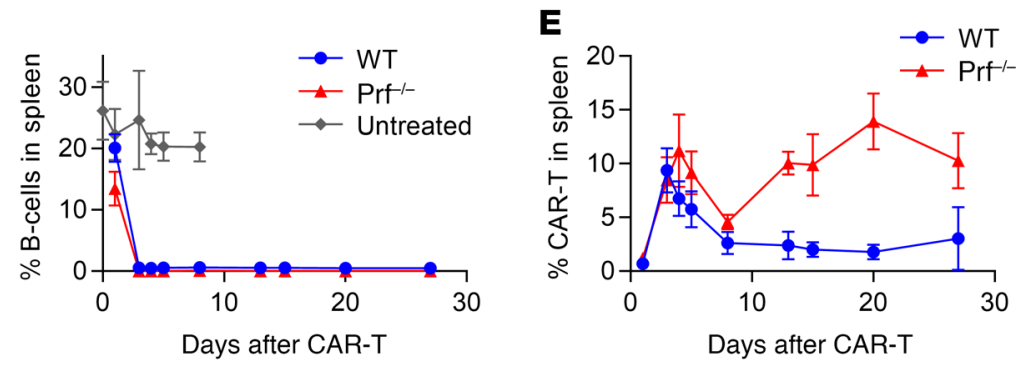

G

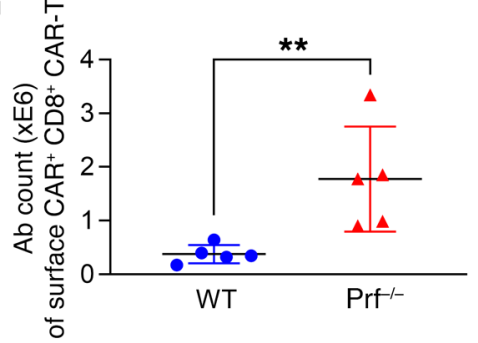

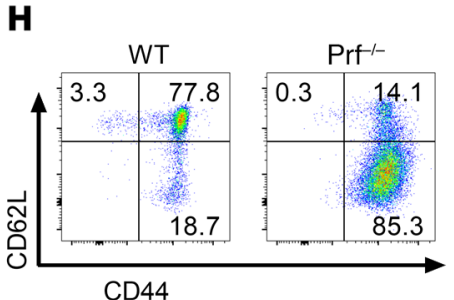
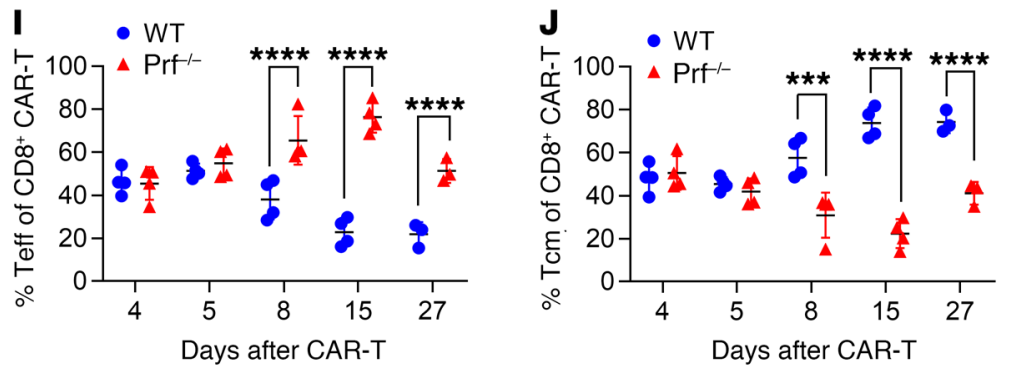

$\mathbf{K}$

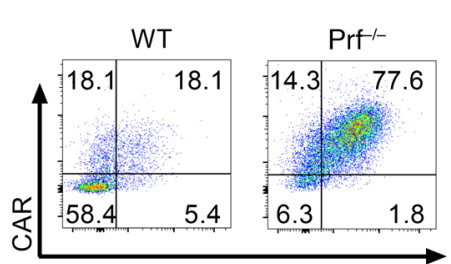

PD-1

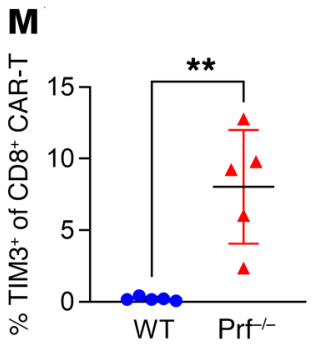

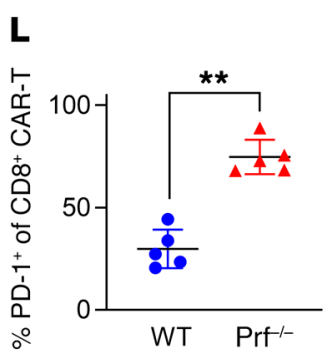

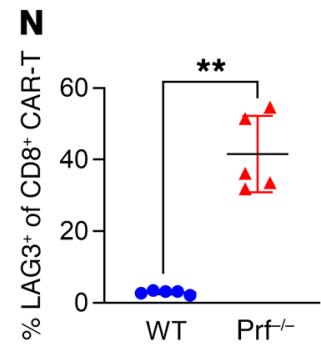


Figure 3. Prf ${ }^{-1}$ CAR T cell undergo a late reexpansion in the absence of detectable antigens. Leukemia-bearing B6-CD45.1 mice were treated as depicted in Figure 2A and received either WT or Prf ${ }^{-1-}$ CAR T cells (CD45.2+) on day 0 at the indicated doses (A and B) or $5 \times 10^{6}$ cells (C-N). (A) The percentages of CD8 ${ }^{+}$CAR T cells (CD45.2 $\left.2^{+} \mathrm{CD} 8^{+}\right)$within total splenocytes were evaluated on day 14. (B) Surface CAR expression on CD8+ CAR T cells was assessed by protein L/streptavidin-PE staining on day 14. (C) Leukemia (CD45.2+CD19+) in BM and (D) B cells (CD19+B220+) in spleens were measured at baseline and at the indicated time points following adoptive T cell transfer. (E) The percentages of adoptively transferred CAR T cell $\left(\right.$ CD45.2 $\left.2^{+} \mathrm{CD3}^{+}\right)$in spleens and (F) surface CAR expression on CD8 ${ }^{+}$CAR T cell in spleens were monitored at indicated time points. (C) The absolute (Abs.) number of CD8 ${ }^{+}$CAR T cells expressing surface CAR in spleens was evaluated on day 14. (H-J) The composition of $\mathrm{CD} 44^{-} \mathrm{CD} 62 \mathrm{~L}^{+}$naive, $\mathrm{CD} 44^{+} \mathrm{CD} 62 \mathrm{~L}^{+}$ Tcm, and CD44 ${ }^{+} \mathrm{CD} 2 \mathrm{~L}^{-}$Tem or Teff cells within the CD8 ${ }^{+}$CAR T cell subset in spleens was assessed. (H) Representative dot plots (day 14) and the percentages of (I) Teff and (J) Tcm cells in CD8+ CAR T cells are shown. (K-N) Expression of surface CAR, PD-1, TIM3, and LAC3 on CD8 ${ }^{+}$CAR T cells in spleens were assessed by flow cytometry on day 14. (K) Representative dot plots and the percentages of WT and $\mathrm{Prf}^{-{ }^{-}-\mathrm{CD} 8}{ }^{+}$CAR T cells expressing (L) PD-1, (M) TIM3, and (N) LAG3 are shown. Data are reported as the mean \pm SD (A-G, I, J, and $\mathbf{L}-\mathbf{N})$. $n=5$ (A, B, G, and $\mathbf{L}-\mathbf{N}) ; n=4$ (C-F, I, and J). Figures are representative of 3 replicate experiments. ${ }^{*} P<0.05$, ${ }^{* *} P<0.01$, ${ }^{* * *} P<$ 0.001 , and ${ }^{* * *} P<0.0001$, by 1 -way ANOVA with Šidák's correction (A, B, I, and J) or Mann-Whitney $U$ test ( $\mathbf{G}$ and $\mathbf{L}-\mathbf{N}$ ).

ure 4, E and F). Furthermore, recipient-derived $\mathrm{CD} 8^{+} \mathrm{T}$ cells were skewed away from a naive $\mathrm{T}$ cell phenotype and toward a Tcm or Teff phenotype in $\mathrm{Prf}^{-/}$CAR T cell recipients (Figure 4, G and H). We observed no significant changes in the phenotype of recipientderived $\mathrm{CD} 4^{+} \mathrm{T}$ cells (Supplemental Figure 6). These changes in recipient-derived immune-associated cells suggest that the lack of perforin not only directly affected CAR T cells but also induced a proinflammatory microenvironment that indirectly affected other immune cells, potentially through soluble mediators.

Indeed, Prf ${ }^{-/}$CAR T cell recipients had increased expression of multiple proinflammatory cytokine genes and circulating cytokine levels. Evaluation of a panel of inflammatory genes in whole BM from CAR T cell-recipient mice revealed 9 genes that were differentially expressed $(P<0.01)$ in $\mathrm{Prf}^{-/-}$CAR T cell recipients compared with WT CAR T cell recipients (Figure 4I). Of these, 7 genes, including Nod2, Il1b, and Tlr2, were involved in inflammatory responses and positive regulation of cytokine secretion (Figure 4I). Levels of $I l 1 b$ (pro-IL-1 $\beta$ ) and Il18 mRNA transcripts in whole BM from Prf ${ }^{-/}$CAR T cell recipients were significantly higher than those in WT CAR T cell recipients on day 8 (Figure 4, J and K). Il33 mRNA was only detected at very low levels in both $\mathrm{Prf}^{-/}$and WT CAR T cell groups (Supplemental Figure 7A). Next, we measured the levels of multiple cytokines in the serum of CAR T cellrecipient mice (Figure 4, L-N, and Supplemental Figure 7, B-L). Consistent with in vitro experiments, IFN- $\gamma$ was differentially elevated in $\mathrm{Prf}^{-/}$compared with WT CAR T cell-recipient mice on day 3 , corresponding to the initial maximal in vivo CAR $\mathrm{T}$ cell expansion (Figure 4L). During the Prf $^{-/}$CAR T cell reexpansion phase (days 13-20), we found that TNF- $\alpha$ levels were significantly higher in $\mathrm{Prf}^{-/}$CAR T cell recipients (Figure 4M). IL-6 levels were not differentially elevated in $\mathrm{Prf}^{-/}$CAR T cell recipients compared with the levels in their WT counterparts throughout the treatment course (Figure $4 \mathrm{~N}$ ). Thus, perforin-deficient CAR T cells upregulate a complex proinflammatory cascade including increased cir- culating IFN- $\gamma$ levels, upregulation of IL-1 family members, and secondary inflammatory changes reminiscent of gene expression signatures reported in HLH and MAS (30-32).

Cotransfer of WT CAR T cells does not prevent late expansion of Prf/- CAR T cells. Although detectable leukemia cells and B cells were rapidly cleared after Prf $^{-/-}$CAR T cell treatment (Figure 3, C and D), residual antigens below the detection limit could hypothetically drive $\operatorname{Prf}^{-/}$CAR T cell reexpansion, particularly given the less efficient antigen clearance by $\operatorname{Prf}^{-/-}$CAR $T$ cells (Figure 1C and Figure 2, B-E, G, and H). We hypothesized that unmeasured residual antigen should be cleared by coinfusion of WT CAR T cells and Prf $^{-/}$CAR T cells, thus preventing late Prf $^{-/}$ CAR T cell expansion if it was a response to an antigen burdendependent process. Alternatively, if Prf $^{-/}$CAR T cells induced an inflammatory environment independently of the residual antigen, then $\mathrm{Prf}^{-/}$CAR T cells would be predicted to potentially alter the phenotype of coadministered WT-CAR T cells. To differentiate between these hypotheses, $\operatorname{Prf}^{-/}$CAR T cells were coinfused with equal numbers of WT CAR T cells or untransduced WT T cells (mock) into leukemia-bearing mice, and the origin of the $\mathrm{T}$ cells was distinguished using congenic markers (Figure 5A and Supplemental Figure 8). When Prf $^{-/}$CAR T cells (Thy1.1 ${ }^{-} \mathrm{CD} 45.2^{+}$) and WT CAR T cells (Thy1.1 ${ }^{+} \mathrm{CD} 45.2^{+}$) were coadministered to leukemia-bearing mice at a 1:1 ratio, the former represented a significantly higher percentage of donor cells on days 7 and 14 after transfer (Figure 5B). Thus, Prf ${ }^{-/}$CAR T cells expanded to a greater extent than did WT CAR T cells in a competitive setting. Furthermore, the frequency of $\operatorname{Prf}^{-/}$CAR T cells with surface CAR expression remained high on day 14, even upon coadministration of WT CAR T cells (Figure 5, C and D). Interestingly, when cotransferred with Prf $^{-/}$CAR T cells, a significantly higher percentage of the WT CAR T cells had surface CAR expression on day 14 compared with the WT CAR T cells infused alone (Figure 5, C and D). These data strongly suggest that $\mathrm{Prf}^{-/}$CAR T cells create an inflammatory milieu that alters the phenotype of perforin-competent CAR T cells. Indeed, the increased Teff to Tcm ratio detected in $\mathrm{Prf}^{-/}$CAR T cell was also detected in $\mathrm{WT} \mathrm{CD}^{+} \mathrm{CAR}$ T cells following their cotransfer (Figure 5, E and F). Collectively, these data demonstrate the critical role of $\mathrm{Prf}^{-/}$CAR T cells in inducing an environment that alters the phenotype of host immune cells as well as adoptively transferred CAR T cells that harbor physiological levels of perforin.

$H L H$-like toxicities in patients receiving anti-CD22 CAR T cells. HLH-like manifestations have been reported as a complication of anti-CD19 CAR T cell therapy, although the incidence of this toxicity profile is not fully established $(10,33-35)$. We recently reported our clinical experience with anti-CD22 CAR T cells with a $4-1 \mathrm{BB}$ costimulatory domain (16). In this trial, among 50 patients with CRS, 19 (38\%) subsequently developed HLH-like toxicities defined by published criteria (see "Supplemental Text 2 " in the supplemental materials and ref. 16). HLH-like manifestations were associated with highly elevated ferritin (Table 1) and a constellation of inflammatory responses (16) including the presence of hemophagocytosis in BM (Figure 6A). An HLH-like manifestation typically occurred as a secondary inflammatory response following CRS, presenting as a biphasic wave of inflammation, whereas patients who had only CRS had 1 peak of cytokine eleva- 
A

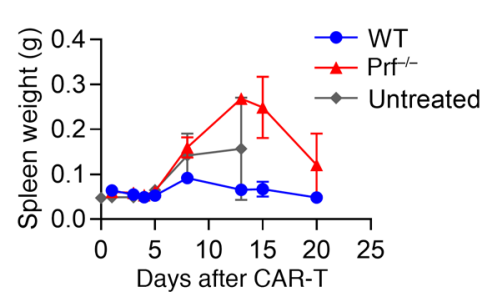

B

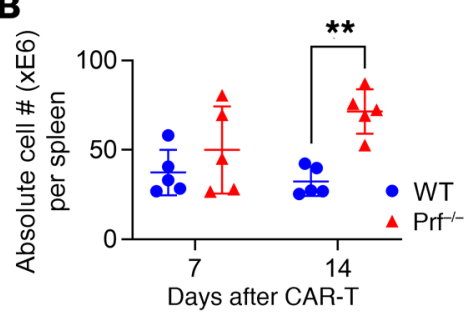

C

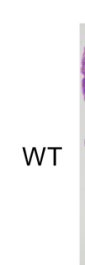

$\mathrm{Prf}^{\prime-}$
$(4 \times)$

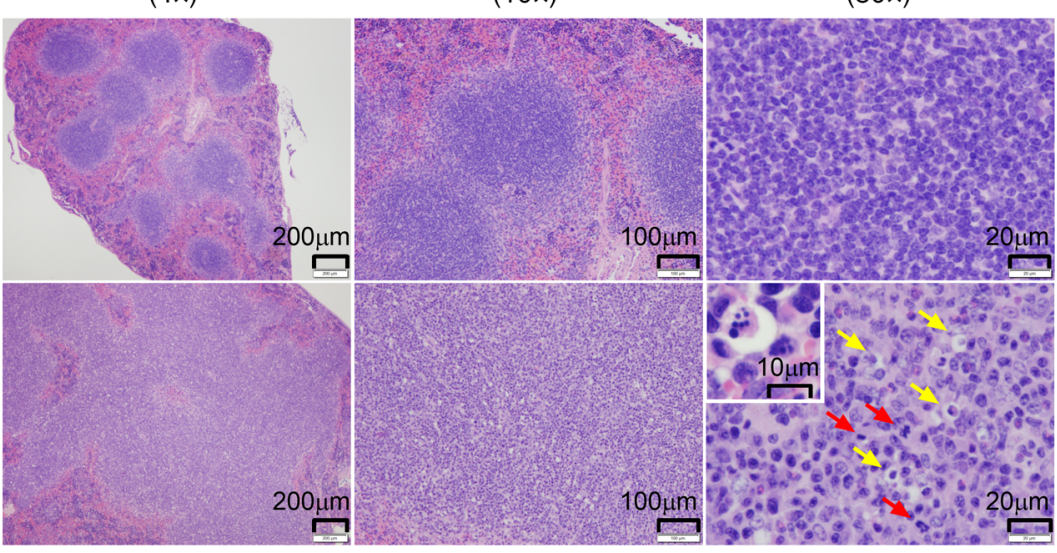

D

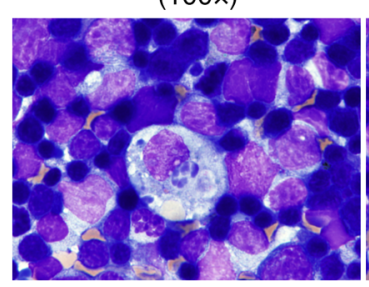

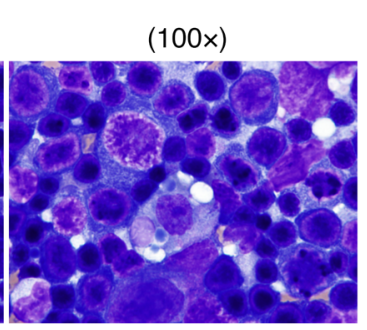

E

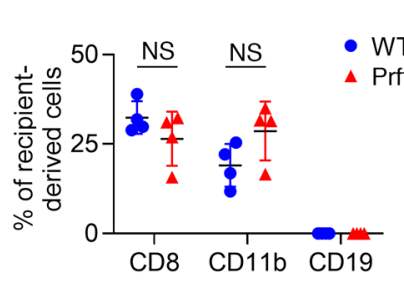

$\mathbf{F}$

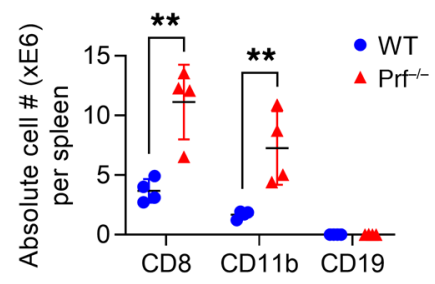

G

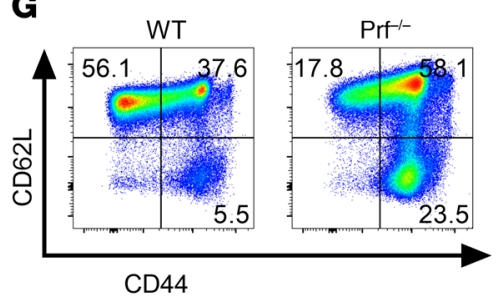

J

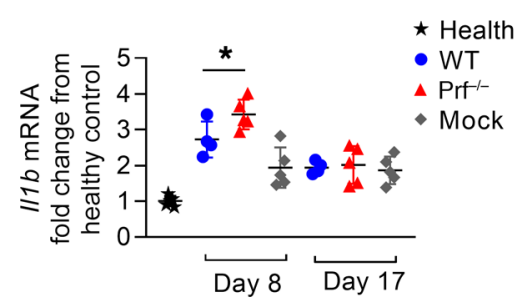

$\mathbf{L}$

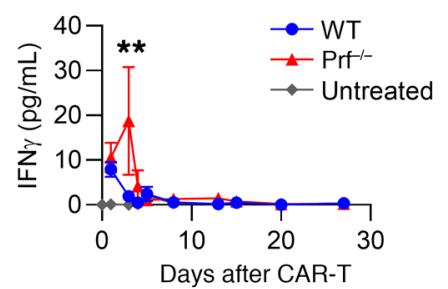

H

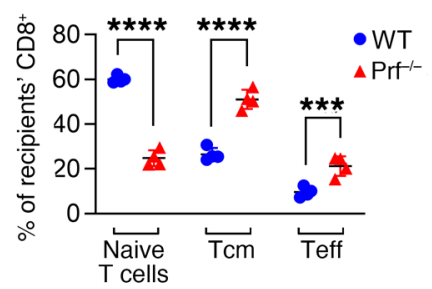

$\mathbf{K}$
I

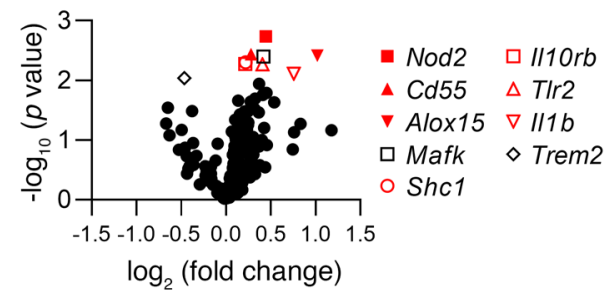

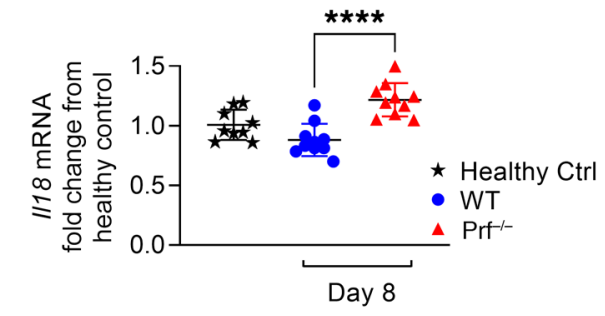

M

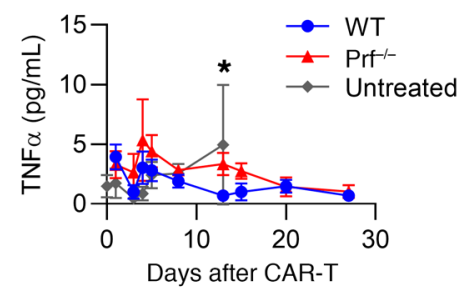

$\mathbf{N}$

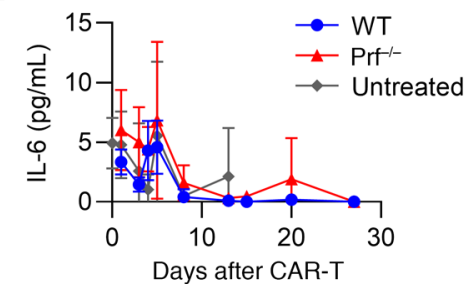


Figure 4. Prf ${ }^{-1-}$ CAR T cells induce inflammation in recipients. Leukemiabearing mice were treated with CAR T cells $\left(5 \times 10^{6}\right.$ cells) as depicted in Figure 2A. (A) The weight of the recipients' spleens and (B) absolute total splenocyte counts were monitored. (C) H\&E-stained images of recipients' spleens harvested on day 15. In $\mathrm{Prf}^{-/-}$tissue in far right bottom panel, phagocytes (yellow arrows) and mitotic figures (red arrows) are highlighted. Scale bars: $200 \mu \mathrm{m}, 100 \mu \mathrm{m}$, and $20 \mu \mathrm{m}$ (original magnification, $100 \times$ for the enlarged inset showing a representative phagocyte). (D) Giemsa stain of spleen section touch preparation from $\mathrm{Prf}^{-1-}$ CAR T cell recipients on day 14. Two representative hemophagocytes are shown (original magnification, 100x). (E) The percentages and (F) absolute numbers of recipient-derived (CD45.1 $\left.1^{+} \mathrm{CD} 45.2^{-}\right) \mathrm{CD}^{+}, \mathrm{CD}_{11 \mathrm{~b}^{+} \text {, and CD19+ }}$ cells on day 15 in spleens, as assessed by flow cytometry. (G) Representative dot plots and (H) statistical comparison of CD44-CD62 $\mathrm{L}^{+}$naive T cell, $\mathrm{CD} 44^{+} \mathrm{CD} 62 \mathrm{~L}^{+} \mathrm{Tcm}$ cell, and $\mathrm{CD}_{4}{ }^{+} \mathrm{CD} 62 \mathrm{~L}^{-}$Tem or Teff cell composition within recipient-derived $\mathrm{CD} 8^{+} \mathrm{T}$ cells. (I) Gene expression in whole BM from recipients (NanoString nCounter Inflammation panel). Volcano plot shows gene expression data comparing recipients of Prf $^{-1-}$ CAR T cells with those of WT CAR T cells $\left[-\log _{10}(P\right.$ value $)$ vs. $\log _{2}$ (fold change)]. Genes with a differential expression of $P<0.01$ are annotated in the legend. Genes involved in inflammatory pathways are further highlighted in red. (J and $\mathbf{K}$ ) Expression levels of (J) pro-IL-1 $\beta$ and (K) IL-18 were assessed by RT-qPCR. (L-N) Serum levels of (L) IFN- $\gamma$, (M) TNF- $\alpha$, and (N) IL-6 in CAR T cell recipients were measured at the indicated time points using the Cytokine Beads Array kit. Data are reported as the mean $\pm \mathrm{SD}(\mathbf{A}, \mathbf{B}, \mathbf{E}, \mathbf{F}, \mathbf{H}$, and J-N). $n=5$ (B and I); $n=4(\mathbf{A}, \mathbf{E}, \mathbf{F}, \mathbf{H}$, and $\mathbf{L}-\mathbf{N}) ; n=$ 4-5 (J); $n=9-10$ (K). Figures are representative of 3 replicate experiments (A-H and $\mathbf{J}-\mathbf{N}$ ). ${ }^{*} P<0.05,{ }^{* *} P<0.01,{ }^{* *} P<0.001$, and ${ }^{* * * *} P<0.0001$, by Kruskal-Wallis test with Dunn's correction (B and $\mathbf{M}$ ) or 1-way ANOVA with Šidák's correction (E, F, H, J, K, and L). Ctrl, control.

tion (Figure 6, B and C). The median time to the onset of HLH-like toxicities was 14 days (range, 7-26 days) after CAR T cell infusion, which was approximately 1 week after the onset of CRS; the majority of HLH-like toxicities presented either after typical CRS had resolved or while CRS was resolving. None of the patients developed HLH-like manifestations without first having CRS. As previously reported, the majority of CRSs were of low grade (90\% were grade 1-2), and the severity of CRS did not predict subsequent occurrences of HLH-like manifestations (16).

Patients who experienced HLH-like manifestations had significantly higher percentages of circulating CAR T cells on day 14 , then again on day 28 , compared with those who experienced only CRS and no subsequent HLH-like manifestations; in the latter group, CAR T cells contracted by day 28 (Figure 6D). In serum or plasma, peak levels of multiple cytokines and inflammatory markers, including IFN- $\gamma$, IL-1 $\beta$, and IL-18, were significantly higher among patients with HLH-like toxicities than in those without (Table 1 and Figure 6, E-G). These clinical observations in human anti-CD22 CAR T cell recipients resemble the findings from our murine model, in which HLH-like manifestations were linked to the elevation of multiple inflammatory cytokines and a persistence of $\mathrm{CAR}^{+}$cells well beyond the initial CRS phase.

Impact of IFN- $\gamma$ neutralization and IL-1 blockade on Prf ${ }^{--}$CAR $T$ cell function and inflammation. We explored cytokine axes that could be therapeutically targeted to mitigate HLH-like manifestations in the context of CAR T cell therapy. First, we sought to target IFN- $\gamma$, which was significantly elevated in $\operatorname{Prf}^{-/-}$CAR T cell-recipient mice compared with WT CAR T cell-recipient mice (Figure $4 \mathrm{~L}$ ). IFN- $\gamma$ has been implicated in primary HLH pathophysiology, and the FDA recently approved emapalumab, an IFN- $\gamma$ - neutralizing antibody, for the treatment of refractory HLH (22, 36). Daily treatment with IFN- $\gamma$-neutralizing antibody for 2 weeks following CAR T cell infusion (Figure 7A) had no impact on the initial clearance of leukemia cells or normal B cells (Supplemental Figure 9, A-D), but led to leukemia relapse and worse survival in Prf $^{-/}$CAR T cell-recipient mice (Figure 7B, median survival of 63 days with IFN- $\gamma$ neutralization vs. an undefined median survival in the isotype control), consistent with in vitro cytotoxicity data (Supplemental Figure 9H). This result suggests an important role of IFN- $\gamma$ in CAR T cell-mediated leukemia clearance, particularly when CAR T cells are defective in granule-mediated cytotoxicity. Furthermore, IFN- $\gamma$ neutralization did not inhibit $\mathrm{Prf}^{-/}$CAR T cell reexpansion on day 14 (Figure 7C), and phenotypic changes observed in Prf $^{-/}$CAR T cells were unaltered (Figure 7, D-F) or even exacerbated (Supplemental Figure 9, E-G) in the presence of neutralizing anti-IFN- $\gamma$ monoclonal antibodies.

We next assessed whether IFN- $\gamma$ could drive inflammatory toxicities in a perforin-competent model, using CAR T cells manufactured from ARE-Del mice, which have stabilized Ifng mRNA transcripts and increased IFN- $\gamma$ production due to homozygous or heterozygous deletion of a $162 \mathrm{nt}$ AU-rich element region in the 3 '-UTR of the Ifng gene $(37,38)$. As expected, recipients of AREDel CAR T cells had significantly higher serum IFN- $\gamma$ levels than did WT CAR T cell recipients during the initial in vivo CAR T cell expansion (Figure 7G), which was proportionate to the affected allele number (ARE homozygous vs. heterozygous deletion) and resembled the serum IFN- $\gamma$ kinetics of $\operatorname{Prf}^{-/-}$CAR T cell recipients. However, 2 weeks after infusion, mice receiving ARE-Del CAR $\mathrm{T}$ cells had fewer $\mathrm{CAR}^{+}$cells than did those receiving WT CAR T cells (Figure 7H). ARE-Del CAR T cells did not exhibit an increase in the Teff phenotype or acquisition of PD-1 expression either (Figure 7, I and J). These data collectively suggest that IFN- $\gamma$, during the early CAR T cell expansion phase, is not directly responsible for later Prf $^{-/-}$CAR T cell reexpansion. Furthermore, our data indicate that IFN- $\gamma$ is not an optimal therapeutic target in the context of CAR T cell therapy.

Another group of cytokines implicated in the pathophysiology of HLH and MAS are IL- 1 family members (e.g., IL- $1 \alpha$, IL-1 $\beta$, IL-18, and IL-33) $(18,39-43)$. Indeed, we observed significantly higher levels of serum IL-1 $\beta$ and IL-18 in human CD22 CAR T cell recipients who developed $\mathrm{HLH}$ as compared with those who did not (Figure 6, F and G). IL-1 $\beta$ and IL-18 gene expression was also increased in $\mathrm{Prf}^{-/}$CAR T cell recipients in our murine model (Figure $4, \mathrm{~J}$ and $\mathrm{K}$ ). Therefore, we next sought to assess whether the HLH-like phenotype in $\mathrm{Prf}^{-/-}$CAR T cell recipients could be ameliorated by blocking IL-1 signaling without negatively affecting the antileukemia efficacy of CAR T cells. Anakinra, a recombinant human IL-1R antagonist that is used clinically and cross-reacts with murine IL-1R1 (44), was administered to leukemia-bearing mice treated with WT or Prf ${ }^{-/}$CAR T cells (Figure 8A). Notably, administration of anakinra did not decrease the initial leukemia clearance or survival of mice receiving either WT or $\mathrm{Prf}^{-/}$CAR T cells (Figure 8, B and C), indicating that IL-1 was not critical for CAR T cell effector function. Although anakinra treatment did not alter the increased frequency of $\mathrm{CD} 8^{+} \mathrm{CAR} \mathrm{T}$ cells, surface $\mathrm{CAR}^{+}$ cells, or Teff predominance of $\mathrm{Prf}^{-/}$CAR T cells at a late time point (Figure 8, D-F), it reduced the levels of other circulating proin- 
A

WT donor:

Thy $1.1^{+} /$CD $45.2^{+}$
Recipients:

Thy1.1-/CD45.2-/CD45.1+

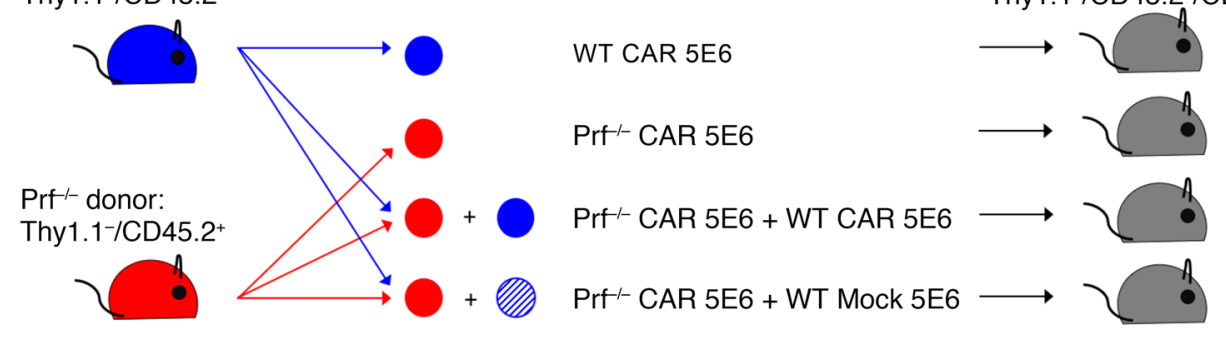

B
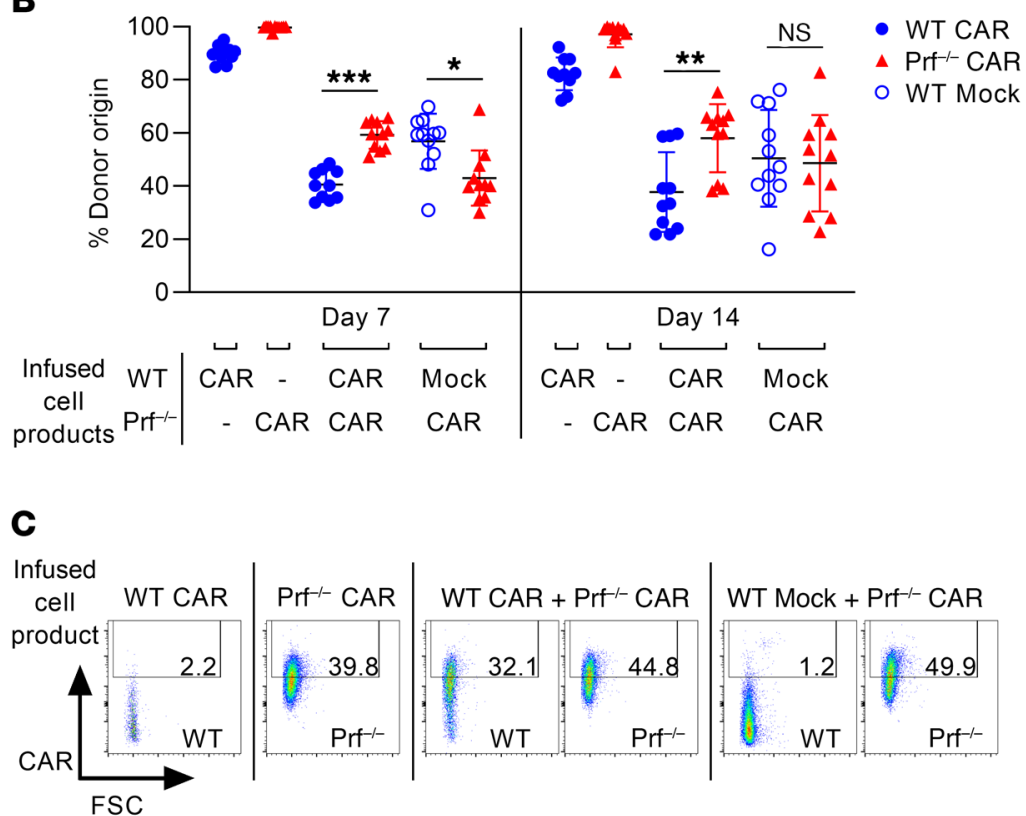

D

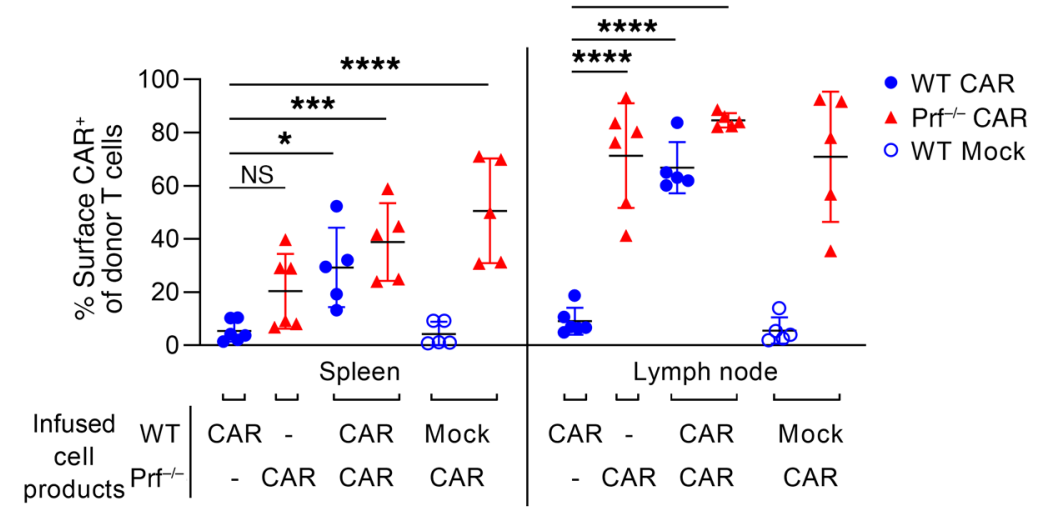

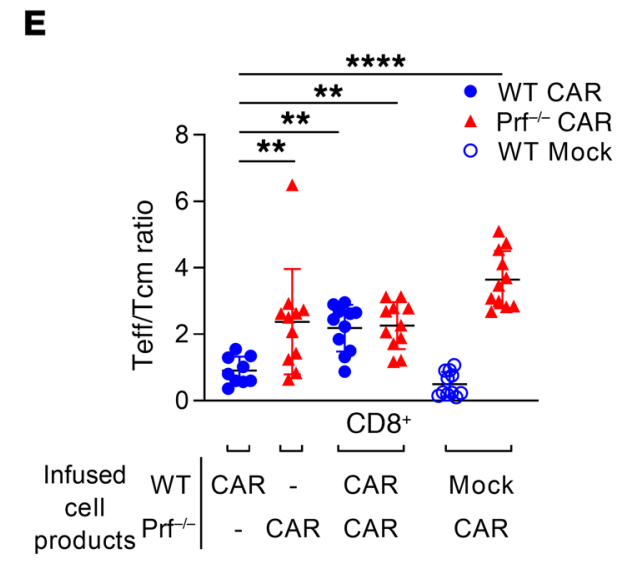

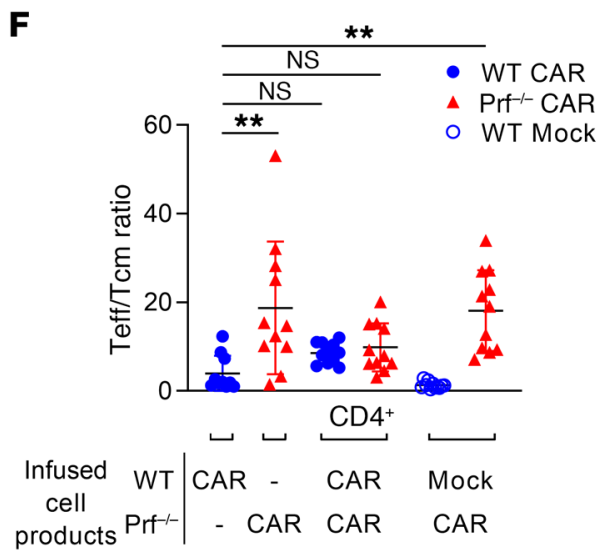

$\mathbf{F}$ $\begin{gathered}\text { cell } \\ \text { products }\end{gathered} \mathrm{Prf}^{--} \mid$- CAR CAR CAR

Figure 5. The reexpansion of Prf ${ }^{-/-}$CAR T cells is not inhibited by coinfusion of WT CAR T cells. (A) Leukemia-bearing mice were lymphodepleted with cyclophosphamide according to the treatment scheme depicted in Figure 2A. To distinguish between adoptively transferred CAR T cell subsets, the following congenic strains were used: B6-Thy1.1 for WT CAR T cells and WT mock (Thy1.1 $1^{+}$CD 45.2 $2^{+}$CD45.1-), B6-Prf1-KO for Prf ${ }^{-{ }^{-}}$CAR T cells (Thy1.1 $1^{-}$ CD45.2+CD45.1-), and B6-CD45.1 for recipients (Thy1.1-CD45.2-CD45.1+). Leukemia-bearing mice were treated with WT CAR T cells (5 $\times 10^{6}$ cells), Prf ${ }^{-1-}$ CAR T cells $\left(5 \times 10^{6}\right.$ cells), a 1:1 mixture of Prf- ${ }^{-}$CAR T cells and WT CAR T cells $\left(5 \times 10^{6}\right.$ each, for a total of $10 \times 10^{6}$ cells $)$, or a 1:1 mixture of Prf- ${ }^{-1-}$ CAR T cells and WT untransduced T cells (Mock) $\left(5 \times 10^{6}\right.$ each, for a total of $10 \times 10^{6}$ cells). (B) Donor origin of adoptively transferred T cells was determined by Thy 1.1 expression on the CD45.2 subset (gating strategy is described in Supplemental Figure 8). Percentages of WT versus Prf ${ }^{-1-} \mathrm{T}_{\text {cells within the CD45.2 }} 2^{+}$subset in spleens on days 7 and 14 are shown. (C) Surface CAR expression on adoptively transferred T cells was assessed by flow cytometry on day 14 . Representative dot plots from spleen samples are shown. FSC, forward scatter. (D)Percentages of donor T cells with cell-surface CAR expression in spleens and lymph nodes on day 14. (E and $\mathbf{F})$ Teff/Tcm cell ratios within adoptively transferred (E) CD8 ${ }^{+}$and $(\mathbf{F}) \mathrm{CD}^{+}$subsets on day 14 . Data are reported as the mean \pm SD $(\mathbf{B}$ and $\mathbf{D}-\mathbf{F}) . n=5-6(\mathbf{D}) ; n=9-11$ (B, E, and $\mathbf{F}$, pooled from independent 2 experiments). Figures are representative of 2 replicate experiments. ${ }^{*} P<0.05,{ }^{* *} P$ $<0.01,{ }^{* *} P<0.001$, and ${ }^{* * *} P<0.0001$, by Kruskal-Wallis test with Dunn's correction (B and $\mathbf{F}$ ) or 1-way ANOVA with Šidák's correction (D and E). 
Table 1. Peak levels of ferritin and cytokines in serum/plasma during the first 28 days after CAR T cell infusion

\begin{tabular}{|c|c|c|c|}
\hline $\begin{array}{l}\text { Ferritin }(\mu \mathrm{g} / \mathrm{L}) \text { and } \\
\text { Cytokines }(\mathrm{pg} / \mathrm{mL})\end{array}$ & $\begin{array}{l}\text { No HLH }{ }^{A} \text {, median } \\
(25 \%-75 \% \text { IQR) }\end{array}$ & $\begin{array}{c}\text { HLH }^{\mathrm{A}}, \text { median } \\
(25 \%-75 \% \text { IQR })\end{array}$ & $P$ value \\
\hline Ferritin ${ }^{B}$ & $22,758(3,554-52,686)$ & $206,740(171,968-420,273)$ & $<0.0001$ \\
\hline $\mathrm{IFN}-\gamma$ & $352.2(196.7-1041)$ & $2800(1,838-2,900)$ & $<0.0001$ \\
\hline IL-1 $\beta$ & $0.77(0.45-2.09)$ & $3.51(1.02-48.95)$ & 0.001 \\
\hline $\mathrm{IL}-2$ & $1.32(0.79-3.51)$ & $2.89(1.15-5.54)$ & 0.05 \\
\hline IL-4 & $0.46(0.12-3.17)$ & $1.36(0.57-4.70)$ & 0.03 \\
\hline IL-6 & $41.58(18.83-214.5)$ & $904.5(264.1-1,480)$ & $<0.0001$ \\
\hline IL-10 & $55.94(22.02-154)$ & $338.7(128.1-567.4)$ & 0.0001 \\
\hline IL-12p70 & $0.43(0.25-0.98)$ & $1.96(0.82-5.62)$ & 0.0007 \\
\hline IL-18 & $0.77(0.45-2.09)$ & $3.51(1.023-8.91)$ & $<0.0001$ \\
\hline TNF- $\alpha$ & $12.77(9.17-23.62)$ & $27.1(16.2-43.91)$ & 0.002 \\
\hline GM-CSF & $1.12(0.79-4.53)$ & $3.43(1.15-9.89)$ & 0.07 \\
\hline MIP-1 $1 \alpha$ & $105.7(67.12-180.1)$ & $223.8(157-422.2)$ & 0.0001 \\
\hline
\end{tabular}

${ }^{A}$ Analyses were restricted to those patients who had CRS; patients without CRS were not included in this table. One-sided $P$ values were determined by Mann-Whitney $U$ test. ${ }^{B}$ For ferritin, $n=19$ patients (no $\mathrm{HLH}$ ) and $n=18$ patients $(\mathrm{HLH})$, given the initial lack of monitoring of ferritin in this trial. For IL-18, data were available for $n=24$ patients (no HLH) and $n=17$ patients (HLH). For all other cytokines, $n=27$ patients (no HLH) and $n=18$ patients (HLH).

and NK cells, whereas $\mathrm{CD} 4^{+} \mathrm{T}$ cells use alternative mechanisms (45). Interestingly, we have previously noted that CAR-stimulated $\mathrm{CD} 4^{+} \mathrm{T}$ cells acquire a "CD8-like" gene expression profile compared with TCR stimulation (26). Additional studies of other known effector mechanisms (e.g., Fas/Fas-L, TRAIL) may reveal distinct mechanisms by which CAR-activated $\mathrm{T}$ cells exert cytotoxicity compared with TCR-activated T cells $(46,47)$.

Prior animal studies of CAR T cell-mediated toxicities have mainly focused on modeling CRS and/or neurotoxicity, and none has specifically focused on HLH-like manifestations. In addition to a nonhuman primate model of anti-CD2O CAR T cells and CRS (48), other groups have reported murine xenograft models of CRS (49-51) as more accessible animal models. However, interpretation of these xenograft models is confounded by xenogeneic graft-versushost disease (xeno-GVHD) that hampers the evaluation of long-term outcomes. Although an impressive humanized mouse model has been developed that is nonxenoreactive and successfully circumvented xeno-GVHD manifestations in the context of CAR T cell therapy (52), such models still fail to recapitulate

flammatory cytokines including IFN- $\gamma$, TNF- $\alpha$, and IL-27 (Figure $8 \mathrm{G})$. Thus, blockade of a single cytokine may not completely reverse the HLH-like phenotype, but anakinra treatment can decrease multiple proinflammatory cytokines without negatively affecting antileukemia effector function of CAR T cells.

\section{Discussion}

We and others have observed that CAR T cell therapy is associated with a spectrum of cytokine-mediated inflammatory toxicities that involve complex interactions between CAR T cells and recipient immune cells $(9,10,12,16)$. Despite the severity and high incidence rate of these toxicities, there are substantial gaps in our understanding of the mechanisms, particularly for late HLH-like manifestations. In this study, we used a murine model of perforindeficient CAR T cells and demonstrated a link between poor CAR T cell granule-mediated cytotoxicity and subsequent secondary inflammation in recipient mice. We found that perforindeficient CAR $\mathrm{T}$ cells underwent delayed reexpansion in the absence of detectable antigen and could trigger an HLH-like phenotype, resembling a clinical manifestation occurring in human CAR T cell recipients.

An additional aspect of these findings is the specific contribution of the effector mechanisms used by CAR T cells, mechanisms that have not been well studied to date, particularly in vivo. We demonstrated that CAR $\mathrm{T}$ cells could mediate antileukemia activity despite the absence of perforin, although with reduced potency. One compensatory mechanism may be IFN- $\gamma$-mediated responses, as our data showed that IFN- $\gamma$ neutralization negatively affected the ability of $\operatorname{Prf}^{-/}$CAR T cells to eradicate leukemia in vitro and in vivo. The dependence of $\mathrm{CD} 4^{+} \mathrm{CAR} T$ cells on perforin was surprising, as it is widely accepted in T cell receptor (TCR) settings that perforin-mediated target killing plays an important role in the cytotoxic function of $\mathrm{CD}^{+}$cytotoxic $\mathrm{T}$ lymphocytes the full scope of interactions expected between adoptively transferred CAR T cells and the recipient's immune system. In contrast, using an entirely syngeneic murine system, we showed that CAR $\mathrm{T}$ cells defective in cytotoxic function cause an array of secondary inflammatory changes in recipient-derived cells. Interestingly, in a prior syngeneic murine model of second-generation anti-CD19 CAR T cells (53), cytokine-driven chronic toxicities were observed only in $\mathrm{BALB} / \mathrm{c}$ recipients, and not in $\mathrm{C} 57 \mathrm{BL} / 6$ or $\mathrm{C} 3 \mathrm{H}$ mice, suggesting there are host factors that play a role in toxicity establishment. In the current study, we did not observe any toxicities in C57BL/6 recipients receiving WT CAR T cells, but we were able to induce HLH-like phenotypes in recipient mice by using perforinknockout $\mathrm{T}$ cell donors.

We chose to investigate granule-mediated cytotoxicity, specifically perforin, in CAR T cells because of perforin's well-established pathogenetic link to primary $\operatorname{HLH}(19,20)$. Although not completely elucidated, a mechanistic association between the lack of perforin and a hyperinflammatory state has been extensively studied in TCR settings, leading to multiple plausible hypotheses $(20,21,54-59)$. In our model, the only cell population lacking perforin was the CAR T cell, in contrast to primary HLH models in which perforin-deficient recipients (or deficiency in other genes in granule-mediated cytotoxicity pathways) are used. Despite this, the effect of perforin deficiency was not limited to CAR T cells but rather led to secondary effects in host-derived cells, suggesting the presence of proinflammatory mediators induced by perforindeficient CAR T cells. In fact, our in vitro cytokine measurements as well as gene expression profiling of $\mathrm{Prf}^{\text {/- }}$ CAR T cells confirmed that an array of proinflammatory mediators were upregulated. In a well-studied primary HLH model that uses LCMV infection in perforin-deficient hosts, IFN- $\gamma$ secreted by antigen-specific CD ${ }^{+}$ $\mathrm{T}$ cells has been implicated as the major driver of HLH pathology (22). Indeed, emapalumab, an IFN- $\gamma$-neutralizing monoclonal 
A

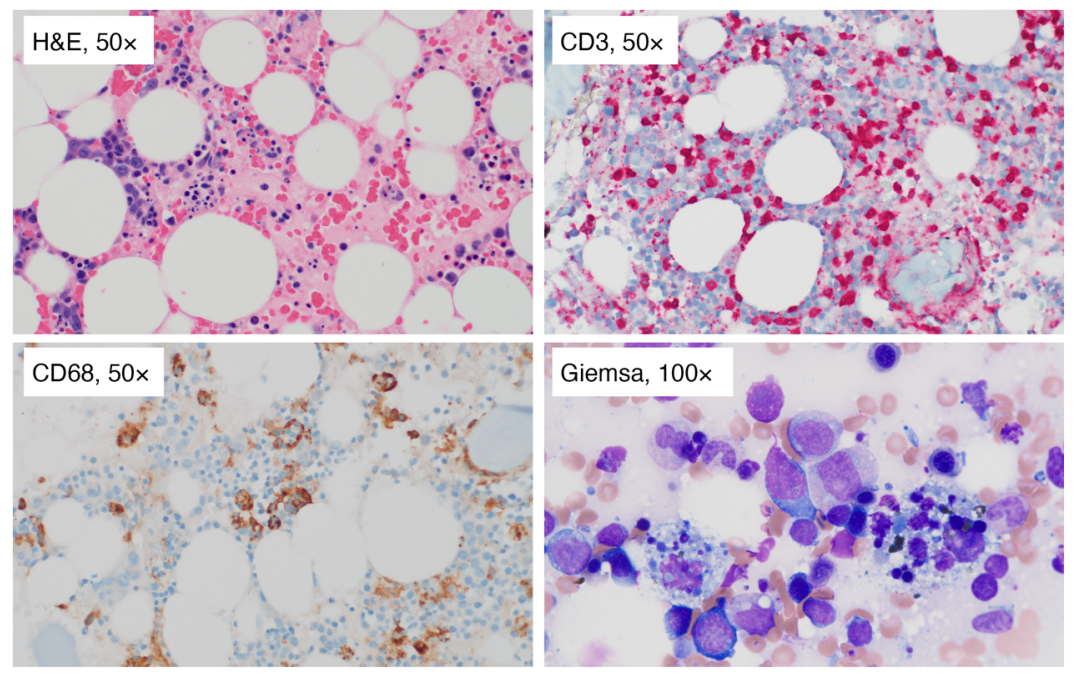

B

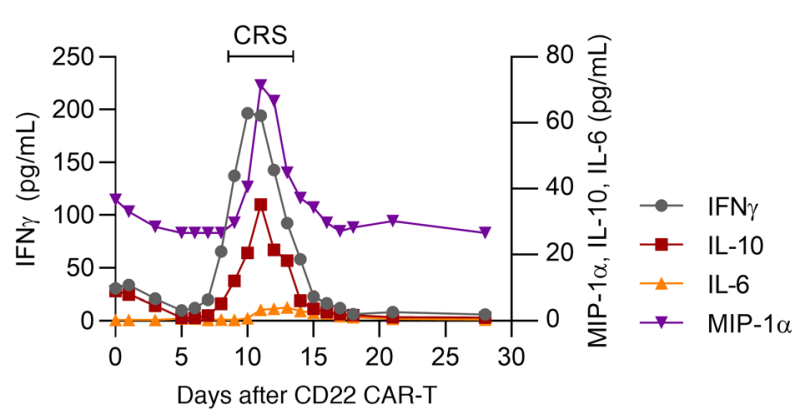

C

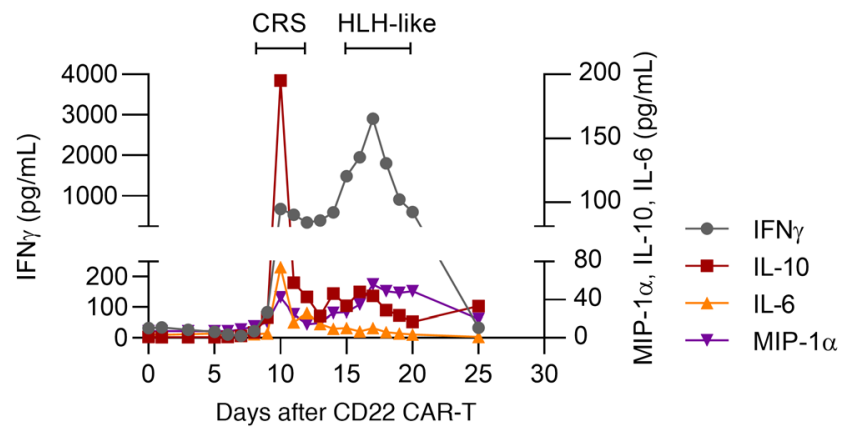

D

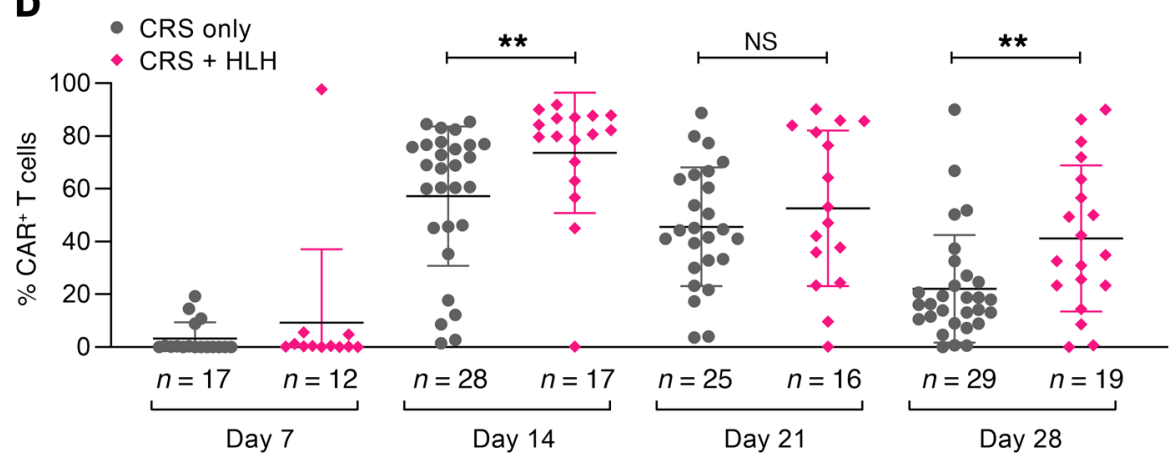

E

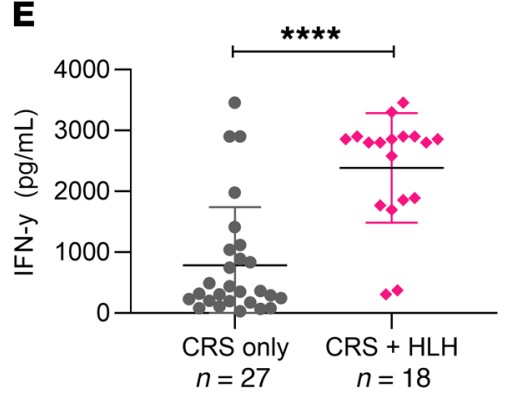

$\mathbf{F}$

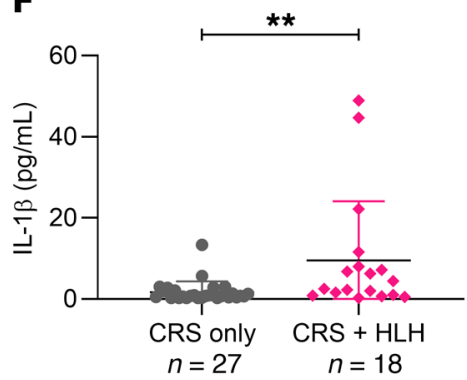

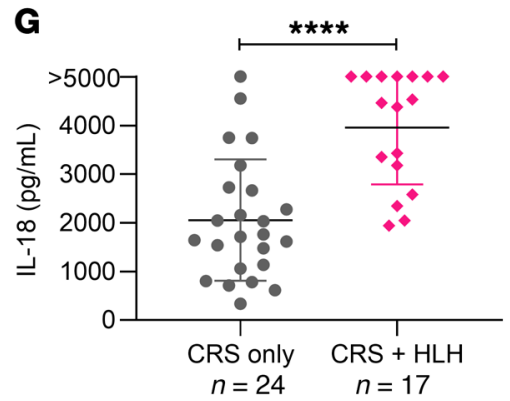


Figure 6. HLH-like manifestations associated with increased circulating CAR T cells were observed in anti-CD22 CAR T cell recipients. (A) BM samples obtained on day 28 after CAR T cell infusion. H\&E-stain shows decreased trilineage hematopoiesis with increased macrophages. CD3 immunohistochemical (IHC) stain highlights extensive T cell infiltration with flow cytometric confirmation of anti-CD22 CAR positivity in 59\% of T cells. CD68 IHC stain highlights hemophagocytic macrophages. Giemsa stain of BM aspirate also shows hemophagocytosis. Original magnification, $50 \times$ (H\&E, CD3, CD68 stains) and 100× (Giemsa stain). (B) Representative chronological changes in serum cytokine levels from patient 52 who had CRS without subsequent HLH. (C) Representative chronological changes in serum cytokine levels from patient 37 who had CRS and subsequent HLH. (D) The percentages of circulating T cells $\left(\mathrm{CD}^{+}\right)$that stained positive for surface CAR expression were assessed by flow cytometry at the indicated time points. (E-G) Peak levels (during the first 28 days) of (E) IFN- $\gamma$, (F) IL-1 $1 \beta$, and (C) IL-18 in serum/plasma. Data shown in D-C include all patients who were diagnosed with CRS according to previously published criteria (9), and patients who had never been diagnosed with CRS are not included. Data were stratified according to the presence or absence of HLH diagnosis (in addition to CRS) after CAR T cell infusion. Data are reported as the mean \pm SD (D-C). ${ }^{*} P<0.05,{ }^{* *} P<0.01,{ }^{* *} P<0.001$, and ${ }^{* * *} P<0.0001$, by Mann-Whitney $U$ test $(\mathbf{D}-\mathbf{G})$.

antibody, has been shown to be efficacious in the treatment of patients with primary HLH (36). Our data demonstrated that IFN- $\gamma$ elevation in Prf ${ }^{/-}$CAR T cell recipients was not driving the HLH-like phenotype and, in fact, may have been "protective" against late non-antigen burden-dependent CAR T cell proliferation and hyperactivation. This is not entirely surprising, given that prior studies have shown that IFN- $\gamma$ and other inflammatory factors can influence subsequent $\mathrm{T}$ cell contraction and memory differentiation in TCR-activated T cell settings (60-63). Moreover, IFN- $\gamma$ neutralization led to early leukemia relapses in the absence of granule-mediated cytotoxicity, implying that IFN- $\gamma$ was involved in the CAR T cell therapeutic effect. Further studies are required to fully address the relative contribution of IFN- $\gamma$ to CAR T cell efficacy and toxicity.

Accumulating published data implicate pathophysiological roles of the IL-1 cytokine family (e.g., IL-1 $\alpha$, IL-1 $\beta$, IL-18, and IL-33) in HLH and MAS, a clinical syndrome similar to HLH $(18,39-43)$. Other investigators have also shown that IL-1 derived from recipients' myeloid cell populations contributes to CAR T cell-induced CRS and neurotoxicity using xenogeneic and xenotolerant murine models $(50,52)$. In both our murine model and human clinical experience, IL-1 $\beta$ and IL-18 axes were associated with HLH-like manifestations. Blockade of the IL-1 axis alone in our murine model did not reverse the phenotype. This is perhaps not surprising, given the concurrent elevation of numerous other proinflammatory mediators, suggesting that targeting multiple cytokines (such as IL-1 and IL-18) may be more effective. In the current study, we measured only total IL-18, but future studies investigating the balance between IL-18BP and biologically active free IL-18 will be important, as this cytokine axis has been shown to be critical in MAS in non-CAR settings $(39,42)$. Because of the lack of differential elevation in circulating IL-6 levels or IL-6 gene expression in Prf $^{-}$- compared with WT CAR T cell recipients, we did not explore the IL- 6 axis in the current study. However, further investigation of the IL- 6 axis in our murine model could also be considered, given its well-established role in CRS $(12,14)$. In addition to IL-6, alter- native proinflammatory mediators or processes implicated in the pathophysiology of CRS and neurotoxicity include GM-CSF (64), endothelial activation induced by possibly multiple mediators (13), and autocrine effects of catecholamines (65). Another group has also reported the promise of inhibiting the JAK/STAT pathway with ruxolitinib to manage CAR T cell-induced CRS in an antiCD123 CAR T cell murine xenograft model (66). Immune-competent models combined with clinical correlates, as presented here, have the potential to identify combinations of therapeutically targetable cytokines or molecular pathways that mitigate inflammatory toxicities while maintaining CAR T cell efficacy.

We lack genetic data from most of our patients regarding their perforin and other genes implicated in primary HLH (see "Supplemental Text 3" in the supplemental materials). However, given that nearly $40 \%$ of the subjects developed HLH-like manifestations, it is unlikely that these patients are all genetically predisposed to HLH. Our current study does not definitively establish why human CAR T cells that are likely perforin competent can induce HLHlike toxicities similar to those seen with murine perforin-deficient CAR T cells. CAR T cells rely on artificial signaling for activation, and it is logical to speculate that downstream signaling and effector functions of CAR-activated T cells differ from those of TCRactivated $\mathrm{T}$ cells. Thus, it is possible that a nonphysiological overproduction of cytokines by CAR T cells drives the HLH-like phenotype in humans, even in perforin-competent settings. The need for perforin-deficient donor T cells to model HLH-like toxicities may reflect a limitation of the syngeneic murine model using mice in a stardard clean facility: the vast majority of nonmodified $\mathrm{T}$ cells in laboratory mice are naive and their myeloid cells are less activated (67), which does not mimic a human who has been exposed to numerous antigens (67-69). Patients with multiply relapsed leukemia also have been exposed to numerous chemotherapies, corticosteroids, and other immunosuppressants, and their T cells are probably dysfunctional (25). We hypothesize that perforindeficient CAR T cells with a decreased ability to control leukemia at early time points may more accurately reflect the human CAR T cells derived from patients who have suboptimal $\mathrm{T}$ cell function.

We found no evidence that HLH-like manifestations occurred in the context of leukemia recurrence in patients who received anti-CD22 CAR T cells (16). The second wave of CAR T cell expansion accompanied by HLH-like manifestations in our murine model also occurred in the absence of detectable antigen, and even coinfusion of WT CAR T cells did not prevent Prf ${ }^{--}$CAR T cell reexpansion. However, we cannot conclude that the reexpansion or HLH-like inflammation is an antigen-independent process, because the recipients have continued B-lymphopoiesis, exposing CAR T cells to antigens even when the recipients are $\mathrm{B}$ cell aplastic. As demonstrated by both our in vitro and in vivo data, Prf $^{\prime-}$ CAR T cells exerted less efficient clearance of leukemia and normal B cells, suggesting that each CAR T cell has theoretically longer exposure to cognate antigen after initial activation. The presence of coinfused WT CAR T cells did not prevent initial activation of $\mathrm{Prf}^{/-}$CAR T cells by antigens. It remains a speculation whether a prolonged per-cell exposure to antigen leads to overactivation of CAR T cells and a resultant proinflammatory response. An inefficient target clearance and increased antigen exposure duration (as measured by prolonged immunological synapse time) 
A

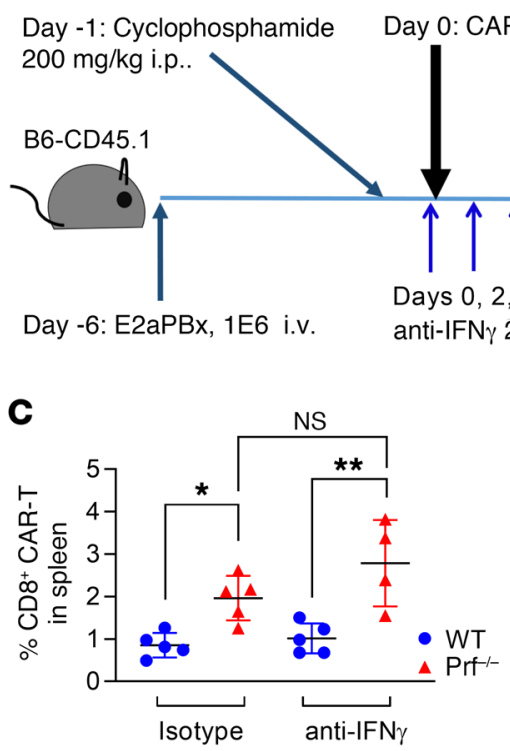

$\mathbf{F}$

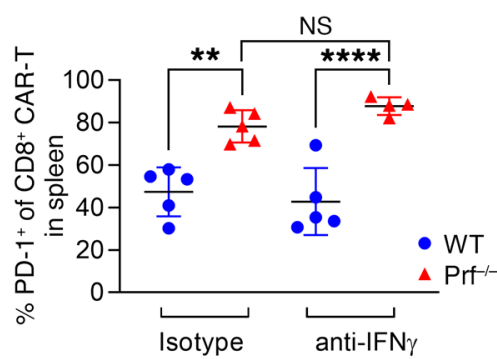

H

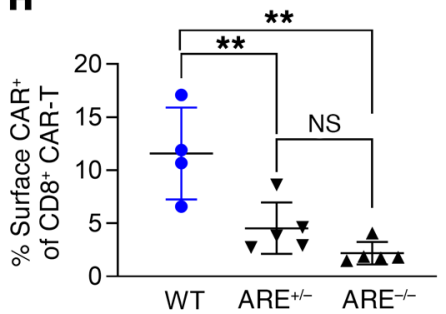

B

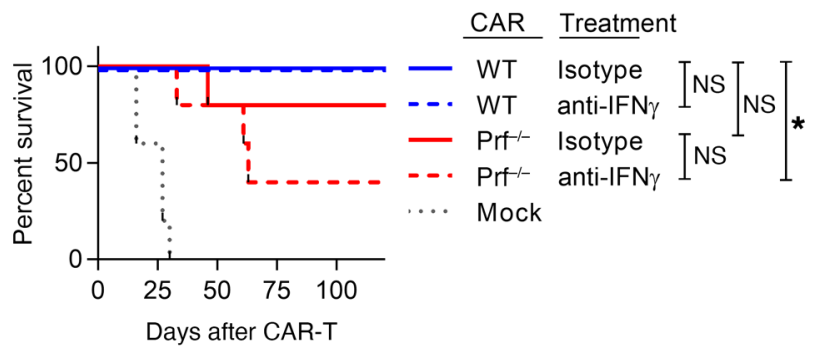

D

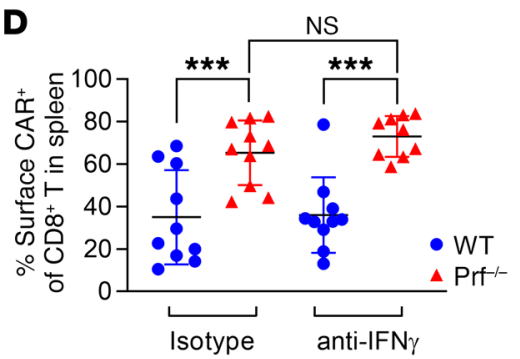

G

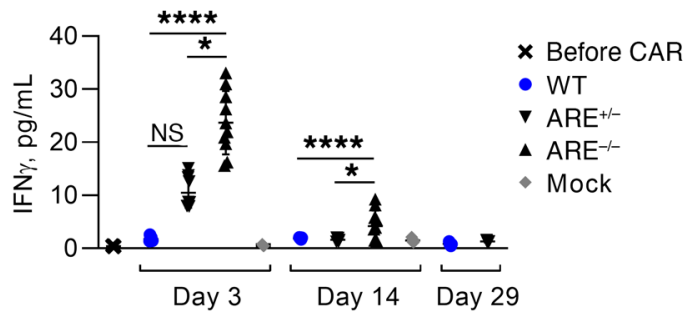

I

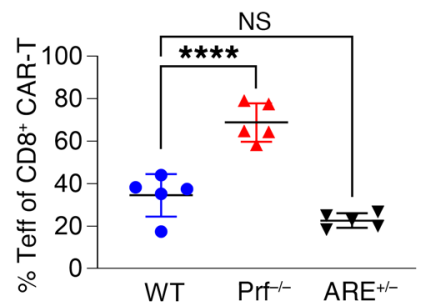

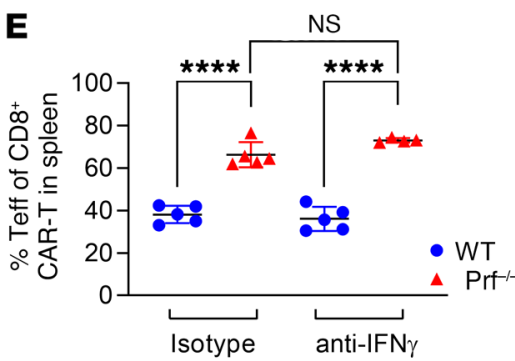

$\mathbf{J}$

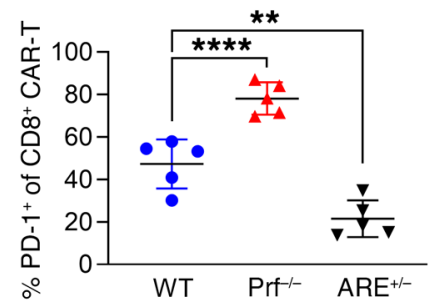

Figure 7. IFN- $\gamma$ neutralization negatively impacts CAR T cell leukemia clearance. (A) Treatment scheme of neutralizing IFN- $\gamma$ in CAR T cell recipients. Mice were treated with i.p. injection of an IFN- $\gamma$-neutralizing antibody (XMG1.2, $200 \mu \mathrm{g}$ ) or an isotype control (rat IgG1, 200 $\mu \mathrm{g}$ ) on days 0, 2, 4, 6, 8, 10, and 12. Some mice were euthanized on day 13, which was 24 hours after the last administration of antibodies, while the rest were kept for survival analyses. (B) KaplanMeier survival curve. (C) The percentages of CD8+ CAR T cells in spleens on day 13 were assessed by flow cytometry. (D) Surface CAR expression, (E) the percentages of CD44+CD62L- (Tem or Teff) cells, and (F) PD-1 expression among adoptively transferred CD8 $8^{+}$CAR T cell (CD45.2 $2^{+} / C D 8^{+}$) subsets in spleens on day 13 were analyzed by flow cytometry. (G-J) Leukemia-bearing mice were treated with CAR T cells $\left(5 \times 10^{6}\right)$ derived from WT, Prf ${ }^{-1}$, or ARE-Del (homozygous- or heterozygous-KO) mice according to the treatment scheme in Figure 2A. (C) Serum IFN- $\gamma$ was measured on day 0 (before adoptive T cell transfer) and on days 3,14 , and 29 after adoptive T cell transfer using the Meso Scale Discovery U-PLEX kit. (H) Surface CAR expression, (I) the percentages of CD44+CD62L- (Tem or Teff) cells, and (J) PD-1 expression within the CD8 ${ }^{+}$CAR T cell subset (CD45.2+CD8 $)$in spleens on day 13 were analyzed by flow cytometry. Data are reported as the mean \pm SD (C-J). $n=4-5(\mathbf{B}, \mathbf{C}, \mathbf{E}, \mathbf{F}$, and $\mathbf{H}-\mathbf{J}) ; n=9-10$ ( $\mathbf{D}$ and $\mathbf{G})$. Figures are representative of 3 replicate experiments. ${ }^{*} P<0.05,{ }^{* *} P<0.01,{ }^{* * *} P<0.001$, and ${ }^{* * * *} P<0.0001$, by log-rank (Mantel-Cox) test (B), 1-way ANOVA with Šidák's correction (C-F and $\left.\mathbf{H}-\mathbf{J}\right)$, or Kruskal-Wallis test with Dunn's correction (G).

have been linked to the overproduction of proinflammatory cytokines by T cells in TCR settings (56). The common denominator of excessive proinflammatory responses seen in CAR T cell settings may also be how potent the CAR T cell's cytotoxicity is relative to how resistant the leukemia is.

In our anti-CD22 CAR T cell trial, neurotoxicity was mostly mild (70), unlike the experience with CD19 CAR T cells (4). CRS was also mostly low grade (grades 1 and 2 ) and well tolerated $(1,16)$. Despite this, 19 of 50 patients who experienced CRS subsequently developed HLH-like manifestations. It is unknown whether CRS and HLH-like toxicities are on the same continuum of the hyperinflammatory response or if each stems from a distinct pathophysiology. It is noteworthy that inflammation can occur in a biphasic manner after anti-CD22 CAR T cell therapy, with the 
A

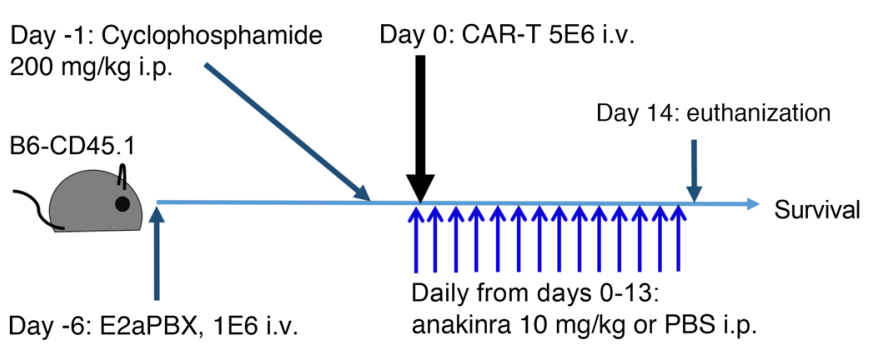

B

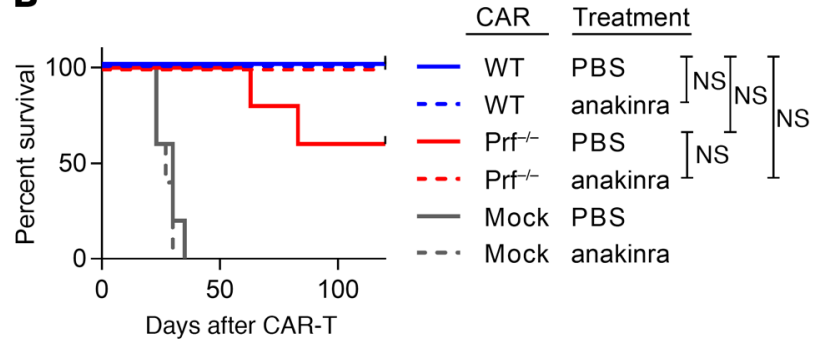

C

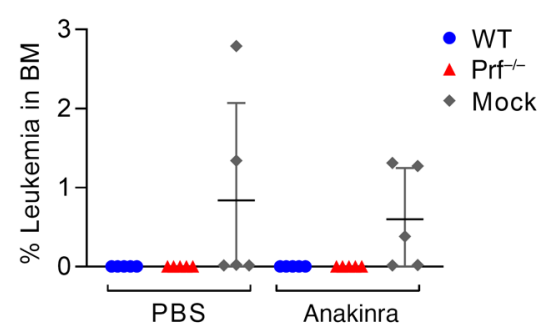

$\mathbf{E}$

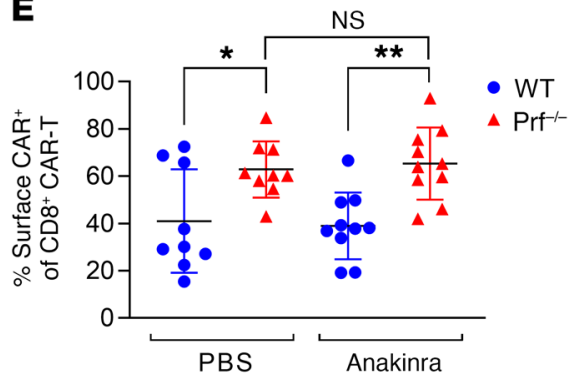

D

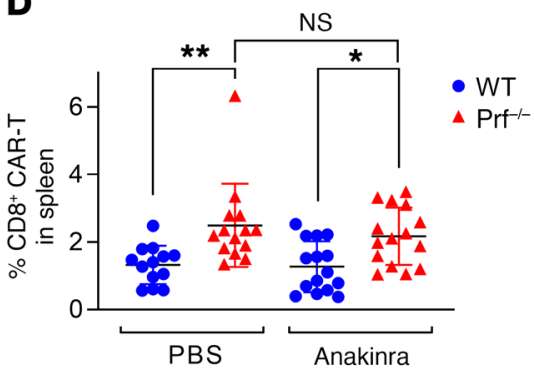

$\mathbf{F}$

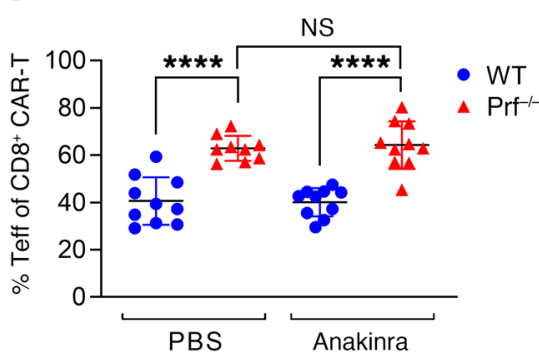

G
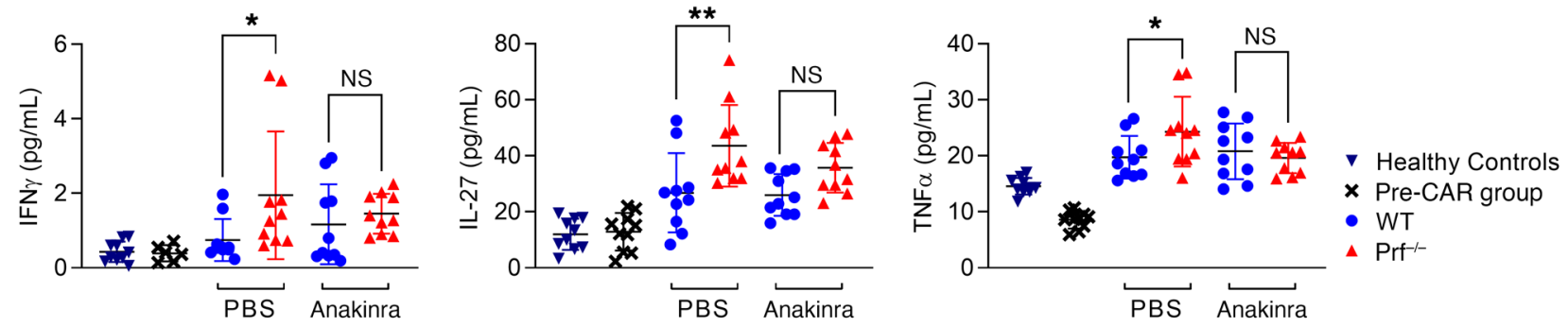

Figure 8. IL-1 blockade improves circulating proinflammatory cytokine levels without inhibiting CAR T cell leukemia clearance. (A) Treatment scheme for IL-1 blockade in CAR T cell recipients. Mice were treated with a daily i.p. injection of anakinra ( $10 \mathrm{mg} / \mathrm{kg} /$ day in $200 \mu \mathrm{L})$ or PBS ( $200 \mu \mathrm{L})$ from days 0 to 13. Some cohorts of mice were euthanized on day 14, which was 24 hours after the last administration of anakinra or PBS, while the rest were kept for survival analyses. (B) Kaplan-Meier survival curve. (C) Leukemia (CD45.2+CD19+) in BM on day 14 was assessed by flow cytometry. (D) Percentages of CD8 ${ }^{+}$CAR T cell in spleens on day 14. (E) Surface CAR expression and (F) percentages of CD44+CD62L- (Tem or Teff) cells within the CD8 ${ }^{+}$CAR T cell subset $\left(C D 45.2^{+} \mathrm{CD}^{+}\right)$in spleens were evaluated on day 14. (C) Serum levels of IFN- $\gamma, \mathrm{IL}-27$, and TNF- $\alpha$ were measured on day 0 (before CAR T cell infusion) and on day 14 using the Meso Scale Discovery U-PLEX kit. Healthy controls were age- and sex-matched untreated littermates. Data are reported as the mean \pm SD (C-C). $n=5$ (B and C); $n=13-15$ (D, data were pooled from independent 2 experiments); $n=9-10$ (E and $\mathbf{F}) ; n=10$ (G, except for $n=6$ in the IFN- $\gamma$ pre-CAR group). Figures are representative of 2 replicate experiments. ${ }^{*} P<0.05,{ }^{* *} P<0.01,{ }^{* * *} P<0.001$, and ${ }^{* * * *} P<0.0001$, by log-rank (Mantel-Cox) test (B) or 1-way ANOVA with Šidák's correction (D-G).

first peak of inflammation corresponding to the typical CRS manifestations and the second being HLH-like manifestations. Several groups have proposed prediction models of severe CRS from anti-CD19 CAR T cell therapy (11-13), but each prediction model differs, suggesting the complexity of CRS and the possible need to address toxicity pathogenesis in a cellular product-specific manner. Most reports on CAR T cell toxicities have not made clear distinctions between CRS and HLH-like toxicities (10), perhaps because severe CRS and HLH-like manifestations can chronologically coincide and phenotypically overlap in other cellular products. Therefore, it is difficult to ascertain the actual frequencies of HLH-like manifestations following CAR T cell therapies or compare toxicities between different cellular products. It is interesting to speculate that a CAR targeting an antigen expressed at low den- 
sity (such as CD22) may result in slower kinetics of antigen clearance, prolonged CAR $\mathrm{T}$ cell activation, and a higher likelihood of late inflammatory toxicities.

Limitations of our study are rooted in inherent biological differences between a syngeneic murine system and humans. In our murine model, manifestations of inflammatory toxicities were not associated with measurable clinical symptoms (e.g., weight loss, decreased activity score) or mortality as compared with clinical symptoms in patients. Accordingly, many parameters pathognomonic for HLH-like toxicities in humans, such as serum ferritin elevations, hepatic enzyme elevations, and coagulopathy, were not consistently observed in our murine model. We chose late reexpansion of hyperactivated CAR T cells as a proxy for delayed inflammation in cytokine neutralization experiments, because the self-limiting nature of CAR T cell-induced inflammation in mice restricted our ability to otherwise measure the effects of interventions. Although we did not systematically evaluate IL- 6 as a therapeutic target, the data suggest that IL-6 may be less important in the settings of HLH than in CRS. Another limitation is the fact that the CAR constructs were not equalized between our murine model and the clinical trial: the murine CAR targets murine CD19 and has a murine CD28 costimulatory domain connected with CD3z with partially inactivated immunoreceptor tyrosine-based activation motifs (ITAMs) (27), while our human anti-CD22 CAR in the clinical trial targets human CD22 and has a human 4-1BB costimulatory domain with intact CD3z. The effects of these structural differences in CAR were not investigated in the current study. Nonetheless, our data link biphasic CAR T cell kinetics and associated inflammation culminating in HLH-like phenotypes, in both our murine model and humans, and provide opportunities to study mechanisms in order to identify therapeutic targets.

In summary, we have demonstrated that perforin contributes to, but is not essential for, CAR T cell cytotoxicity and is involved in the regulation of secondary inflammatory responses. To our knowledge, this is the first syngeneic murine model of CAR T cell-induced, late-onset inflammatory toxicities that resemble the manifestations of HLH. Our study has important translational implications for further understanding of the pathophysiology of CAR T cell therapy toxicities. This is a critical issue to be addressed as cellular therapy is extended to additional target antigens and diseases in which the spectrum of toxicities may differ from those associated with wellestablished anti-CD19 CAR T cells against B cell malignancies.

\section{Methods}

Mice. C57BL/6 and B6-CD45.1 (B6.SJL-Ptprc ${ }^{\mathrm{a}}$ Pepc $\mathrm{b} /$ BoyJ) mice were purchased from The Jackson Laboratory and the NCI Grantee Program of Charles River Laboratories via the NCI Animal Production Program (Frederick, Maryland, USA). Prf1-KO mice (C57BL/6-Prf1 1 tm1sdz/J) and B6-Thy1.1 (B6.PL-Thy1 1 CyJ) were purchased from The Jackson Laboratory. IFN- $\gamma$ ARE-Del mice on a C57BL/6 background were generated as previously described $(37,38)$. All experiments were performed using female mice between 7 and 12 weeks of age.

Tumor cell lines. The E2aPBX murine pre-B ALL cell line was derived from transgenic mice bearing the human E2a:PBX1 transgene

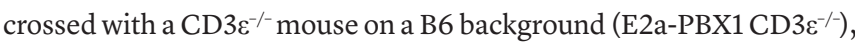
provided by Janetta Bijl (Université de Montréal, Montreal, Canada)
(71). E2aPBX cells were adapted for culture as a stable cell line expressing pre-B ALL markers, including CD19, as previously described (24, 25). As a negative control for in vitro assays, a CD19- $\mathrm{E} 2 \mathrm{aPBX}$-derived cell line was made by editing the CD19 locus using the CRISPR/Cas9 system (23). All cell lines were cultured in $10 \%$ complete mouse media (CMM) containing RPMI 1640 with 10\% heat-inactivated FCS, glutamine (Gibco, Thermo Fisher Scientific, GlutaMAX diluted to $1 \times$ ), nonessential amino acids (Gibco, Thermo Fisher Scientific, MEM-NEAA diluted to $1 \times)$, sodium pyruvate $(1 \mathrm{mM})$, HEPES $(15 \mathrm{mM})$, penicillin (100 units $/ \mathrm{mL})$, streptomycin $(100 \mu \mathrm{g} / \mathrm{mL})$, and 2-mercaptoethanol $(50 \mu \mathrm{M})$. Cell lines tested negative for mycoplasma.

Generation of murine anti-CD19 CAR T cells. The original construct of murine anti-CD19 CAR with the CD28 costimulatory domain and inactivating mutations on the first and third ITAMs of the CD3z, expressed in the mouse stem cell virus-based splice-gag vector (MSGV) retroviral backbone, was provided by James Kochenderfer (NCI, NIH, Bethesda, Maryland, USA) (27). The 293GP cell line was transfected as previously described $(25,26)$ and adapted to make an anti-CD19-CAR stable producer line. Whole splenocytes harvested from euthanized mice were depleted of RBC using ACK Lysing Buffer (Lonza) and enriched for $\mathrm{CD}^{+} \mathrm{T}$ cells using the Mouse $\mathrm{CD}^{+} \mathrm{T}$ cell Enrichment Column (R\&D Systems). For CD4 ${ }^{+}$ or $\mathrm{CD}^{+}$purified CAR $\mathrm{T}$ cell generation, $\mathrm{CD} 4^{+}$or $\mathrm{CD} 8^{+} \mathrm{T}$ Cell Isolation Kits (Miltenyi Biotec) were used, respectively. Isolated $\mathrm{T}$ cells were activated with Dynabeads Mouse T-Activator CD3/CD28 (Life Technologies, Thermo Fisher Scientific) using a 1:1 bead/cell ratio and cultured in CMM in the presence of recombinant human IL-2 (rhIL-2) $(30 \mathrm{IU} / \mathrm{mL})$ and rhIL-7 $(10 \mathrm{ng} / \mathrm{mL})$. On the second and third days of $\mathrm{T}$ cell activation, plates coated with retronectin (Takara Bio Inc.) were spun with retroviral supernatant from the CD19-CAR stable producer cell line. Activated $\mathrm{T}$ cells were then added to the retronectin-coated plate and incubated at $37^{\circ} \mathrm{C}$ for transduction. On the fourth day, T-Activator CD3/CD28 beads were magnetically removed, and transduced $\mathrm{T}$ cells were expanded in culture for 24 to 48 hours before use for in vitro and in vivo studies and for 96 hours for in vitro gene expression profiling experiments.

Adoptive CAR T cell therapy. Recipient B6-CD45.1 mice (CD45.1 $1^{+}$ were injected with E2aPBX cells $\left(1 \times 10^{6}\right.$ cells, CD45.2 $2^{+} 6$ to 14 days before adoptive $\mathrm{T}$ cell transfer. Mice were treated with cyclophosphamide $(200 \mathrm{mg} / \mathrm{kg})$ i.p. 1 day before adoptive $\mathrm{T}$ cell transfer for lymphodepletion. CAR $\mathrm{T}$ cells or mock $\mathrm{T}$ cells (untransduced activated $\mathrm{T}$ cells) were adoptively transferred on day 0 (doses are specified in the figure legends). For in vivo neutralization of IFN- $\gamma$, either neutralizing anti-IFN- $\gamma$ antibodies $(200 \mu \mathrm{g}$, clone XMG1.2, Bio X Cell) or an isotype controls $(200 \mu \mathrm{g}$, rat IgG1 anti-HRP, Bio X Cell) were injected i.p. on the day of CAR T cell adoptive transfer (immediately before the CAR T cell infusion), and then every other day for a total of 7 doses or until the day of euthanization. For anti-IL-1 treatment, mice were injected i.p. with anakinra (10 mg/kg in $200 \mu \mathrm{L}$ PBS, Sobi, Amgen) on the day of CAR $\mathrm{T}$ cell adoptive transfer (immediately before CAR T cell infusion) and daily for a total of 14 doses.

In vitro and in vivo cytokine assessment. For in vitro cytokine assessment, target cells were cocultured with CAR T cells at an effector-to-target (E:T) ratio of 1:1 $\left(1 \times 10^{5}\right.$ cells each per well) in 96-well round-bottomed plates for the indicated durations. Supernatant was collected and analyzed using the Meso Scale Discovery U-Plex Mouse Custom Multiplex Kit (Meso Scale Diagnostics). Levels of selected 
cytokines were confirmed with a Quantikine ELISA Kit (R\&D Systems, for IFN- $\gamma$, IL-2, IL-6) and with the Cytometric Beads Array (BD Biosciences, for IFN- $\gamma$, TNF- $\alpha$, IL-10, IL-6, IL-4, IL-17A, GM-CSF) following the manufacturers' instructions.

For in vivo cytokine analysis, mice were terminally bled as part of the scheduled euthanization, and serum was separated and frozen at $-80^{\circ} \mathrm{C}$ until cytokine measurement. In vivo cytokine levels, except for IL-18, were measured using Cytometric Bead Array kits (BD Biosciences) and/or a Meso Scale Discovery U-PLEX kit (Meso Scale Diagnostics). Mouse serum IL-18 levels were measured using a Mouse IL-18 ELISA Kit (MBL International).

Antibodies and flow cytometry. The antibodies used for flow cytometric analysis are listed in Supplemental Table 1. Murine antiCD19 CAR detection was performed using biotinylated protein L (Thermo Fisher Scientific) and streptavidin-phycoerythrin (PE) (BD Biosciences) as previously described (72). Human anti-CD22 CAR detection was performed using a CD22-Fc chimera protein (R\&D Systems) as previously described (1). Samples were analyzed on a BD LSR-Fortessa or a BD FACSAria II (BD Biosciences). Data were collected using the FACSDiva and analyzed with FlowJo software, version 10 .

CD107a degranulation assay, cytotoxicity assay, and proliferation assay. See Supplemental Methods for further information.

Reverse transcription and quantitative real-time PCR of pro-IL-1 $\beta$, $I L-18$, and IL-33. See Supplemental Methods for further information.

Microarray and Nanostring: sample preparation and data acquisition. Microarray data are publicly available in the NCBI's Gene Expression Omnibus database (GEO GSE130929). See Supplemental Methods for further information.

Clinical trial. The patients described in this study were all enrolled in a phase I trial (clinicaltrials.gov NCT02315612) testing anti-CD22 CAR T cells (CD22CART) for the treatment of patients with relapsed/ refractory CD22+ leukemia or lymphoma. The CD22CART construct $(73,74)$ and details on the clinical trial's design $(1,16)$ have been previously described. This report incorporates data on all study patients who received CD22CART on-study before November 1, 2018 and through a minimum of 28 days after infusion. All patients had serial measurements of circulating cytokine levels evaluated by ELISA at the Frederick National Laboratory for Cancer Research (FNLCR) during the first month after infusion and other clinical laboratory measurements as indicated. Total IL-18 was measured using the Human IL-18 ELISA Kit (MBL International). CRS was defined and graded by previously published criteria (9), and organ toxicities were graded according to the Common Terminology Criteria for Adverse Events (CTCAE), version 4.03. Resolution of CRS was defined by the absence of fever for more than 24 hours, with a concurrent decline of $50 \%$ of the peak value of $\mathrm{C}$-reactive protein and resolution of systemic symptoms associated with CRS. HLH-like manifestations were captured according to the criteria we have previously reported (see "Supplemental Text 2" in the supplemental materials and ref. 16). Diagnostic NK cell function and genetic testing for HLH were performed in select patients following individual consent for clinical testing, and samples were analyzed at Cincinnati Children's Hospital.

Statistics. Survival of mice was analyzed using the Kaplan-Meier method, and survival among different groups was compared using alogrank (Mantel-Cox) test. For continuous variables, data are presented as the mean $\pm \mathrm{SD}$, and comparisons were made using an unpaired
Student's $t$ test (for 2-group comparisons) or 1-way ANOVA (for comparisons among 3 or more groups) followed by pair-wise comparison. When the assumption of normal distribution did not hold and/or when the assumption of equal variances did not hold for ANOVA, nonparametric methods (Mann-Whitney $U$ test, Kruskal-Wallis test) were used. Two-tailed $P$ values of less than 0.05 were considered significant (except for the clinical cytokine analyses in Table 1, which present 1 -sided $P$ values), and $P$ values were adjusted for multiple comparisons using Šidák's or Dunn's correction. Statistical analyses were performed using GraphPad Prism, version 8, for Windows (GraphPad Software). Analyses of gene expression data were performed as described in the Supplemental Methods in the section "Microarray and Nanostring."

Study approval. All animals were cared for in accordance with protocols approved by the IACUC of the NCI. All patients in the antiCD22 CAR T cell clinical trial provided written informed consent, or parental permission with minor assent was obtained when appropriate. All patients were treated in the Pediatric Oncology Branch of the $\mathrm{NCI}$, and the protocol was approved by the IRB of the NCI and the NIH Recombinant DNA Advisory Committee.

\section{Author contributions}

KI and TJF conceived and designed the research. NNS and TJF conceived, designed, and conducted the clinical trial. KI, MP, and RAEC designed and performed experiments and collected, analyzed, and interpreted the data. CDC and MEK contributed to experimental design and execution. HQ developed the murine ALL cell line and anti-CD19 viral supernatant stable producer cell lines. NT contributed to experimental designs, experiment execution, data interpretation, and data presentation. VSZ and TK contributed to the execution of experiments. NNS, AKO, HS, and BY provided patient care under clinical trials and collected clinical data incorporated in this manuscript. NNS and DAL analyzed the clinical data. HAY developed the ARE-Del mice and provided input during project development and data analysis and interpretation. HL, SK, and JFS performed microarray data analysis. ADF and MAE reviewed and interpreted histopathology samples. KI, NNS, NT, and TJF wrote the manuscript, and all authors revised and approved the manuscript.

\section{Acknowledgments}

This study was funded by the NIH intramural program and with federal funds from the NCI, NIH, under contract number HHSN261200800001E. We thank James Kochenderfer (NCI, $\mathrm{NIH}$ ) for the murine CD19-CD28 CAR construct and John Buckley for assistance with mouse experiments. We thank the Frederick National Laboratory for Cancer Research for assisting with gene expression experiments. We would also like to thank the leaderships of the NHLBI and NCI clinical fellowship programs for providing support and critical feedback. The authors thank all the patients and families for their participation in the clinical trial and acknowledge the members of the care teams at the NIH Clinical Center, who provided ongoing care for the patients treated in this study. The content of this publication does not necessarily reflect the views or policies of the Department of Health and Human Services, nor does mention of trade names, commercial products, or organizations imply endorsement by the US Government. 
Address correspondence to: Kazusa Ishii, Experimental Transplantation and Immunotherapy Branch, Genitourinary Malignancies Branch, Center for Cancer Research, National Cancer Institute, NIH, 10 Center Drive, Building 10, Room 13N240, Bethesda, Maryland 20892, USA. Phone: 240.858-3291; Email: kazusa.ishii@nih.gov. Or to: Nirali N. Shah, Pediatric Oncology Branch, Center for Cancer Research, National Cancer Insti- tute, NIH, 10 Center Drive, Building 10-CRC, Room 1W-5750, Bethesda, Maryland 20892, USA. Phone: 240.760.6199; Email: nirali.shah@nih.gov. Or to: Terry J. Fry, University of Colorado Anschutz Medical Campus and Children's Hospital Colorado, 13123 East 16th Avenue, Mail Stop B115, Aurora, Colorado 80045, USA. Phone: 303.724.7293; Email: terry.fry@ cuanschutz.edu.
1. Fry TJ, et al. CD22-targeted CAR T cells induce remission in B-ALL that is naive or resistant to CD19-targeted CAR immunotherapy. Nat Med. 2018;24(1):20-28.

2. Turtle CJ, et al. Immunotherapy of nonHodgkin's lymphoma with a defined ratio of $\mathrm{CD} 8^{+}$and $\mathrm{CD} 4^{+} \mathrm{CD} 19$-specific chimeric antigen receptor-modified T cells. Sci Transl Med. 2016;8(355):355ra116.

3. Porter DL, Levine BL, Kalos M, Bagg A, June $\mathrm{CH}$. Chimeric antigen receptor-modified T cells in chronic lymphoid leukemia. $N$ Engl JMed. 2011;365(8):725-733.

4. Lee DW, et al. T cells expressing CD19 chimeric antigen receptors for acute lymphoblastic leukaemia in children and young adults: a phase 1 dose-escalation trial. Lancet. 2015;385(9967):517-528.

5. Kochenderfer JN, et al. Chemotherapy-refractory diffuse large B-cell lymphoma and indolent B-cell malignancies can be effectively treated with autologous $\mathrm{T}$ cells expressing an antiCD19 chimeric antigen receptor. JClin Oncol. 2015;33(6):540-549.

6. Kochenderfer JN, et al. Eradication of $\mathrm{B}$-lineage cells and regression of lymphoma in a patient treated with autologous $T$ cells genetically engineered to recognize CD19. Blood. 2010;116(20):4099-4102.

7. Brentjens RJ, et al. CD19-targeted T cells rapidly induce molecular remissions in adults with chemotherapy-refractory acute lymphoblastic leukemia. Sci Transl Med. 2013;5(177):177ra38.

8. Neelapu SS, et al. Axicabtagene ciloleucel CAR T cell-cell therapy in refractory large B-cell lymphoma. NEnglJ Med. 2017;377(26):2531-2544.

9. Lee DW, et al. Current concepts in the diagnosis and management of cytokine release syndrome. Blood. 2014;124(2):188-195.

10. Neelapu SS, et al. Chimeric antigen receptor T-cell therapy - assessment and management of toxicities. Nat Rev Clin Oncol. 2018;15(1):47-62.

11. Hay KA, et al. Kinetics and biomarkers of severe cytokine release syndrome after CD19 chimeric antigen receptor-modified T-cell therapy. Blood. 2017;130(21):2295-2306.

12. Teachey DT, et al. Identification of predictive biomarkers for cytokine release syndrome after chimeric antigen receptor T-cell therapy for acute lymphoblastic leukemia. Cancer Discov. 2016;6(6):664-679.

13. Gust J, et al. Endothelial activation and bloodbrain barrier disruption in neurotoxicity after adoptive immunotherapy with CD19 CAR-T cells. Cancer Discov. 2017;7(12):1404-1419.

14. Teachey DT, et al. Cytokine release syndrome after blinatumomab treatment related to abnormal macrophage activation and amelio- rated with cytokine-directed therapy. Blood. 2013;121(26):5154-5157.

15. Le RQ, et al. FDA Approval summary: tocilizum$\mathrm{ab}$ for treatment of chimeric antigen receptor $\mathrm{T}$ cell-induced severe or life-threatening cytokine release syndrome. Oncologist. 2018;23(8):943-947.

16. Shah NN, et al. CD4/CD8 T-cell selection affects chimeric antigen receptor (CAR) T-cell potency and toxicity: updated results from a phase I anti-CD22 CAR T cell-cell trial. J Clin Oncol. 2020;38(17):1938-1950.

17. Voskoboinik I, Whisstock JC, Trapani JA. Perforin and granzymes: function, dysfunction and human pathology. Nat Rev Immunol. 2015;15(6):388-400.

18. Grom AA, Horne A, De Benedetti F. Macrophage activation syndrome in the era of biologic therapy. Nat Rev Rheumatol. 2016;12(5):259-268.

19. Stepp SE, et al. Perforin gene defects in familial hemophagocytic lymphohistiocytosis. Science. 1999;286(5446):1957-1959.

20. Matloubian M, et al. A role for perforin in downregulating T-cell responses during chronic viral infection. JVirol. 1999;73(3):2527-2536.

21. Lykens JE, Terrell CE, Zoller EE, Risma K, Jordan MB. Perforin is a critical physiologic regulator of T-cell activation. Blood. 2011;118(3):618-626.

22. Jordan MB, Hildeman D, Kappler J, Marrack P. An animal model of hemophagocytic lymphohistiocytosis (HLH): $\mathrm{CD} 8^{+} \mathrm{T}$ cells and interferon gamma are essential for the disorder. Blood. 2004;104(3):735-743.

23. Jacoby $\mathrm{E}$, et al. CD19 CAR immune pressure induces B-precursor acute lymphoblastic leukaemia lineage switch exposing inherent leukaemic plasticity. Nat Commun. 2016;7:12320.

24. Jacoby E, Yang Y, Qin H, Chien CD, Kochenderfer JN, Fry TJ. Murine allogeneic CD19 CAR T cells harbor potent antileukemic activity but have the potential to mediate lethal GVHD. Blood. 2016;127(10):1361-1370.

25. Qin $\mathrm{H}$, et al. Murine pre-B-cell ALL induces T-cell dysfunction not fully reversed by introduction of a chimeric antigen receptor. Blood. 2018;132(18):1899-1910.

26. Yang $Y$, et al. TCR engagement negatively affects $\mathrm{CD} 8$ but not CD4 CAR T cell expansion and leukemic clearance. Sci Transl Med. 2017;9(417):eaag1209.

27. Kochenderfer JN, Yu Z, Frasheri D, Restifo NP, Rosenberg SA. Adoptive transfer of syngeneic $\mathrm{T}$ cells transduced with a chimeric antigen receptor that recognizes murine $\mathrm{CD} 19$ can eradicate lymphoma and normal B cells. Blood. 2010;116(19):3875-3886.

28. Cohnen A, et al. Surface CD107a/LAMP-1 protects natural killer cells from degranulation-associated damage. Blood. 2013;122(8):1411-1418.
29. Rutz S, Wang X, Ouyang W. The IL-20 subfamily of cytokines--from host defence to tissue homeostasis. Nat Rev Immunol. 2014;14(12):783-795.

30. Fall N, et al. Gene expression profiling of peripheral blood from patients with untreated new-onset systemic juvenile idiopathic arthritis reveals molecular heterogeneity that may predict macrophage activation syndrome. Arthritis Rheum. 2007;56(11):3793-3804.

31. Sumegi J, et al. Gene expression profiling of peripheral blood mononuclear cells from children with active hemophagocytic lymphohistiocytosis. Blood. 2011;117(15):e151-e160.

32. Canna SW, et al. An activating NLRC4 inflammasome mutation causes autoinflammation with recurrent macrophage activation syndrome. Nat Genet. 2014;46(10):1140-1146.

33. Oved JH, Barrett DM, Teachey DT. Cellular therapy: immune-related complications. Immunol Rev. 2019;290(1):114-126.

34. Hashmi H, et al. Haemophagocytic lymphohistiocytosis has variable time to onset following CD19 chimeric antigen receptor $\mathrm{T}$ cell therapy. $\mathrm{Br} \mathrm{J}$ Haematol. 2019;187(2):e35-e38.

35. Sandler RD, et al. Diagnosis and management of secondary HLH/MAS following HSCT and CAR-T cell therapy in adults; a review of the literature and a survey of practice within EBMT centres on behalf of the Autoimmune Diseases Working Party (ADWP) and Transplant Complications Working Party (TCWP). Front Immunol. 2020;11:524.

36. Locatelli F, et al. Emapalumab in Children with Primary Hemophagocytic Lymphohistiocytosis. N Engl J Med.2020;382(19):1811-1822.

37. Lin FC, et al. IFN- $\gamma$ causes aplastic anemia by altering hematopoietic stem/progenitor cell composition and disrupting lineage differentiation. Blood. 2014;124(25):3699-3708.

38. Hodge DL, et al. IFN-gamma AU-rich element removal promotes chronic IFN-gamma expression and autoimmunity in mice. JAutoimmun. 2014;53:33-45.

39. Weiss ES, et al. Interleukin-18 diagnostically distinguishes and pathogenically promotes human and murine macrophage activation syndrome. Blood. 2018;131(13):1442-1455.

40. Miettunen PM, Narendran A, Jayanthan A, Behrens EM, Cron RQ. Successful treatment of severe paediatric rheumatic disease-associated macrophage activation syndrome with interleukin-1 inhibition following conventional immunosuppressive therapy: case series with 12 patients. Rheumatology (Oxford). 2011;50(2):417-419.

41. Nigrovic PA, et al. Anakinra as first-line disease-modifying therapy in systemic juvenile idiopathic arthritis: report of forty-six patients from an international multicenter series. Arthritis 
Rheum. 2011;63(2):545-555.

42. Girard-Guyonvarc'h C, et al. Unopposed IL-18 signaling leads to severe TLR9-induced macrophage activation syndrome in mice. Blood. 2018;131(13):1430-1441.

43. Rood JE, et al. ST2 contributes to T-cell hyperactivation and fatal hemophagocytic lymphohistiocytosis in mice. Blood. 2016;127(4):426-435.

44. Petrasek J, et al. IL-1 receptor antagonist ameliorates inflammasome-dependent alcoholic steatohepatitis in mice. J Clin Invest. 2012;122(10):3476-3489.

45. Muranski P, Restifo NP. Adoptive immunotherapy of cancer using CD4(+) T cells. Curr Opin Immunol. 2009;21(2):200-208.

46. Dufva $O$, et al. Integrated drug profiling and CRISPR screening identify essential pathways for CAR T cell-cell cytotoxicity. Blood. 2020;135(9):597-609.

47. Singh N, et al. Impaired death receptor signaling in leukemia causes antigen-independent resistance by inducing CAR T cell-cell dysfunction. Cancer Discov. 2020;10(4):552-567.

48. Taraseviciute A, et al. Chimeric antigen receptor T cell-mediated neurotoxicity in nonhuman primates. Cancer Discov. 2018;8(6):750-763.

49. Ruella M, et al. Kinase inhibitor ibrutinib to prevent cytokine-release syndrome after anti-CD19 chimeric antigen receptor $\mathrm{T}$ cells for $\mathrm{B}$-cell neoplasms. Leukemia. 2017;31(1):246-248.

50. Giavridis T, van der Stegen SJC, Eyquem J, Hamieh M, Piersigilli A, Sadelain M. CAR T cell-induced cytokine release syndrome is mediated by macrophages and abated by IL-1 blockade. Nat Med. 2018;24(6):731-738.

51. van der Stegen SJ, et al. Preclinical in vivo modeling of cytokine release syndrome induced by ErbB-retargeted human T cells: identifying a window of therapeutic opportunity? J Immunol. 2013;191(9):4589-4598.

52. Norelli M, et al. Monocyte-derived IL-1 and IL-6 are differentially required for cytokine-release syndrome and neurotoxicity due to CAR T cells. Nat Med. 2018;24(6):739-748.

53. Cheadle EJ, et al. Differential role of Th1 and Th2 cytokines in autotoxicity driven by CD19-specific second-generation chimeric antigen receptor T cells in a mouse model. J Immunol. 2014;192(8):3654-3665.

54. Kägi D, Odermatt B, Mak TW. Homeostatic regulation of $\mathrm{CD}^{+} \mathrm{T}$ cells by perforin. Eur J Immunol. 1999;29(10):3262-3272.

55. Boissonnas A, et al. Foxp $3^{+} \mathrm{T}$ cells induce perforin-dependent dendritic cell death in tumor-draining lymph nodes. Immunity. 2010;32(2):266-278.

56. Jenkins MR, et al. Failed CTL/NK cell killing and cytokine hypersecretion are directly linked through prolonged synapse time. J Exp Med. 2015;212(3):307-317.

57. Huang JF, et al. TCR-Mediated internalization of peptide-MHC complexes acquired by T cells. Science. 1999;286(5441):952-954.

58. Waggoner SN, Cornberg M, Selin LK, Welsh RM. Natural killer cells act as rheostats modulating antiviral T cells. Nature. 2011;481(7381):394-398.

59. Terrell CE, Jordan MB. Perforin deficiency impairs a critical immunoregulatory loop involving murine CD8(+) T cells and dendritic cells. Blood. 2013;121(26):5184-5191.

60. Harty JT, Badovinac VP. Shaping and reshaping CD ${ }^{+}$T-cell memory. Nat Rev Immunol. 2008;8(2):107-119.

61. Badovinac VP, Tvinnereim AR, Harty JT. Regulation of antigen-specific CD8+ T cell homeostasis by perforin and interferon-gamma. Science. 2000;290(5495):1354-1358.

62. Prlic M, Bevan MJ. Exploring regulatory mechanisms of CD8+ T cell contraction. Proc Natl Acad Sci USA. 2008;105(43):16689-16694.

63. Refaeli Y, Van Parijs L, Alexander SI, Abbas AK. Interferon gamma is required for activa- tion-induced death of T lymphocytes. JExp Med. 2002;196(7):999-1005.

64. Sterner RM, et al. GM-CSF inhibition reduces cytokine release syndrome and neuroinflammation but enhances CAR-T cell function in xenografts. Blood. 2019;133(7):697-709.

65. Staedtke V, et al. Disruption of a self-amplifying catecholamine loop reduces cytokine release syndrome. Nature. 2018;564(7735):273-277.

66. Kenderian SS, et al. Ruxolitinib prevents cytokine release syndrome after CART cell therapy without impairing the anti-tumor effect in a xenograft model. Blood. 2016;128(22):652.

67. Abolins S, et al. The comparative immunology of wild and laboratory mice, Mus musculus domesticus. Nat Commun. 2017;8:14811.

68. Willyard C. Squeaky clean mice could be ruining research. Nature. 2018;556(7699):16-18.

69. Beura LK, et al. Normalizing the environment recapitulates adult human immune traits in laboratory mice. Nature. 2016;532(7600):512-516.

70. Shalabi H, et al. Systematic evaluation of neurotoxicity in children and young adults undergoing CD22 chimeric antigen receptor T-cell therapy. J Immunother. 2018;41(7):350-358.

71. Bijl J, Sauvageau M, Thompson A, Sauvageau G. High incidence of proviral integrations in the Hoxa locus in a new model of E2aPBX1-induced B-cell leukemia. Genes Dev. 2005;19(2):224-233.

72. Zheng Z, Chinnasamy N, Morgan RA. Protein L: a novel reagent for the detection of chimeric antigen receptor (CAR) expression by flow cytometry. J Transl Med. 2012;10:29.

73. Haso W, et al. Anti-CD22-chimeric antigen receptors targeting B-cell precursor acute lymphoblastic leukemia. Blood. 2013;121(7):1165-1174.

74. Long AH, et al. 4-1BB costimulation ameliorates $\mathrm{T}$ cell exhaustion induced by tonic signaling of chimeric antigen receptors. Nat Med 2015;21(6):581-590. 